Ergod. Th. \& Dynam. Sys. (1983), 3, 87-118

Printed in Great Britain

\title{
Bifurcation of an attracting invariant circle: a Denjoy attractor
}

\author{
GLEN R. HALL \\ Mathematics Research Center, 610 Walnut Street, University of Wisconsin, \\ Madison, Wisconsin, 53705
}

(Received 4 October 1982)

\begin{abstract}
We construct an example of a $C^{\infty}$ diffeomorphism of an annulus into itself which has an attracting invariant circle such that the map restricted to this circle has no periodic points and no dense orbits. By studying two parameter families of maps of the plane which undergo Hopf bifurcation, particularly the set of parameter values for which the rotation number is irrational, we see that the above example can be considered as a 'worst case' of the loss of smoothness of an attracting invariant circle without periodic orbits.
\end{abstract}

\section{Introduction}

Let $f_{\mu}: \mathbb{R}^{2} \rightarrow \mathbb{R}^{2}$ be a family of $C^{\infty}$ functions which depend smoothly on the parameter $\mu \in \mathbb{C}$. Suppose for all $\mu \in \mathbb{C}, f_{\mu}(0,0)=(0,0)$ and $D f_{\mu}(0,0)$ has eigenvalues $\mu$ and $\bar{\mu}$. When $|\mu|<1$ the origin is asymptotically stable while when $|\mu|>1$ the origin is a repeller. Provided the higher order terms of $f_{\mu}$ satisfy some conditions and $\mu^{n}$ is bounded away from one for $n=1,2,3,4$, the Hopf bifurcation theorem implies that, as $\mu$ passes from the inside to the outside of the circle $|\mu|=1$, a stable invariant circle bifurcates from the origin $([\mathbf{1 8}],[\mathbf{2 1}],[\mathbf{8}],[\mathbf{2 0}],[16])$. As $|\mu|$ increases, the radius of the invariant circle increases, and examples show that the invariant circle can eventually bifurcate into what is called by some a 'strange attractor' exhibiting 'chaotic' behaviour ([5], [2]). The process by which the attracting invariant circle loses smoothness when its rotation number is rational was studied numerically in [2]. In this case the invariant circle loses smoothness gradually. Of particular interest were the mechanisms by which the invariant circle could stop being $C^{1}$. One of these mechanisms is the development of a heteroclinic tangency for the lift of the map to the projective bundle over $\mathbb{R}^{2},[2]$. This mechanism will be seen to be important when the rotation number is irrational where otherwise the situation is quite different.

The main result of this paper is the construction of an example of a $C^{\infty}$ diffeomorphism of an annulus which has an attracting Lipschitz invariant circle such that the map restricted to this circle is a Denjoy map, i.e. a homeomorphism of the circle with no periodic points and no dense orbits. Similar examples have been constructed by Knill [15], Harrison [10, 11] and Herman [13]. Knill constructs a $C^{\infty}$ diffeomorphism of an annulus with an invariant circle on which the map is 
a Denjoy map. However, the map he constructs is hyperbolic on the invariant circle and so the structure of the map near the invariant circle is very complicated. In particular, the invariant circle is not isolated. Harrison's example is of a $C^{2}$ diffeomorphism with the restriction to an invariant circle being a Denjoy map and this invariant circle is semi-stable. Herman's example is a $C^{3-\varepsilon}$ diffeomorphism of the annulus which is area preserving and which contains an invariant circle on which the map is a Denjoy map. See also [9].

The construction we give can be accomplished $C^{1}$-close to certain maps of the annulus which arise after Hopf bifurcation in even simple (polynomial) maps of the plane. However, since the construction uses a degenerate connection between invariant manifolds in the projective bundle over the annulus, we obtain no other information concerning how often these maps occur. Also, it is unknown whether or not an analytic map of this type exists.

$\S 2$ will be used to fix notation used throughout. In $\S 3$ we review the Hopf bifurcation theorem for two parameter families of maps of the plane, particularly when the rotation number is irrational. In particular, we show that the set of parameter values on which a fixed irrational rotation number is assumed is a Lipschitz curve. $\$ 4$ considers the smoothness of invariant circles with irrational rotation number and in $\S 5$ we construct the example discussed above. $\S 5$ is independent of $\S \S 3,4$.

\section{Notation and definitions}

Let $2 \pi \mathbb{Z}=\{2 \pi n: n \in \mathbb{Z}\}$, and let $\mathbb{T}=\mathbb{R} / 2 \pi \mathbb{Z}$ be the circle with circumference equal to $2 \pi$. Let $A=[0,1] \times \mathbb{T}$. Note that the maps

$$
\begin{aligned}
& \eta_{1}: \mathbb{R} \rightarrow \mathbb{T} ; \quad \theta \rightarrow \theta+2 \pi \mathbb{Z} \\
& \eta:[0,1] \times \mathbb{R} \rightarrow A ; \quad(r, \theta) \rightarrow(r, \theta+2 \pi \mathbb{Z})
\end{aligned}
$$

are smooth (natural) covering maps which can be thought of as coordinate systems on $\nabla$ and $A$ respectively. We will deal with these coordinates extensively and hence we will allow the context to determine whether we are speaking of a map from $\mathbb{T}$ to $\mathbb{T}(A$ to $A)$ or its lift from $\mathbb{R}$ to $\mathbb{R}([0,1] \times \mathbb{R}$ to $[0,1] \times \mathbb{R})$. If $f$ is a map from a space to itself then we will let $f^{n}$ denote the $n^{\prime}$ th iterate of $f$, i.e. $f^{n}=f \circ f^{n-1}$.

Definition. A continuous map $h: \mathbb{T} \rightarrow \mathbb{T}$ is called degree one if

$$
H(x+2 \pi)=H(x)+2 \pi
$$

for any lift $H$ of $h$ and any $x \in \mathbb{R}$.

Definition. If $h: \mathbb{T} \rightarrow \mathbb{T}$ is a degree one homeomorphism then the rotation number of $h$, denoted rot $(h)$, is defined to be

$$
\frac{1}{2 \pi} \lim _{n \rightarrow \infty} \frac{H^{n}(x)}{n}
$$

where $H$ is a lift of $h$ satisfying $H(0) \in[0,2 \pi)$ and $x \in \mathbb{R}$.

Remark. This limit exists and is independent of the choice of $x$ ([19]). 
Definition. Let $h: A \rightarrow A$ be a continuous map. We say $h$ is degree one if

$$
H(r, x+2 \pi)=H(r, x)+(0,2 \pi)
$$

for any lift $H$ of $h$ and any $(r, x) \in[0,1] \times \mathbb{R}$.

Notation. Let

and

$$
\lambda_{1}:[0,1] \times \mathbb{R} \rightarrow[0,1]: \quad(r, \theta) \rightarrow r
$$

$$
\lambda_{2}:[0,1] \times \mathbb{R} \rightarrow \mathbb{R}: \quad(r, \theta) \rightarrow \theta
$$

be the natural projections.

Definition. If $h: A \rightarrow A$ is a continuous, degree one map and $z \in A$ then we define the rotation number of $h$ at $z$, denoted $\operatorname{rot}(h, z)$ to be

$$
\frac{1}{2 \pi} \lim _{n \rightarrow \infty} \frac{\lambda_{2}\left(H^{n}(\bar{z})\right)}{n}
$$

where $H$ is a lift of $h$ with $H\left(\frac{1}{2}, 0\right) \in[0,1] \times[0,2 \pi)$ and $\bar{z} \in[0,1] \times \mathbb{R}$ satisfies $\eta(\bar{z})=z$. If the limit does not exist we say $\operatorname{rot}(h, z)$ does not exist.

Definition. Suppose for some integer $n \geq 0$, the degree one maps $h, g: \mathbb{T} \rightarrow \mathbb{T}$ are $n$-times continuously differentiable. Then define

$$
\begin{aligned}
\|h, g\|_{C^{o}} & =\sup _{\theta \in \mathbb{R}}|H(\theta)-G(\theta)| \\
\|h, g\|_{C^{n}} & =\sup _{\theta \in \mathbb{R}}|H(\theta)-G(\theta)|+\sup _{\substack{\theta \in \mathbb{R} \\
1 \leq i \leq n}}\left|\frac{d^{i} H(\theta)}{d \theta^{i}}-\frac{d^{i} G(\theta)}{d \theta^{i}}\right|,
\end{aligned}
$$

where $H$ and $G$ are lifts of $h, g$ respectively with $H(0), G(0) \in[0,2 \pi)$. Similarly, if $h, g: A \rightarrow A$ are $n$-times continuously differentiable maps then

$$
\begin{aligned}
\|h, g\|_{C^{0}}= & \sup _{(r, \theta) \in[0,1] \times \mathbf{R}}\|H(r, \theta)-G(r, \theta)\| \\
\|h, g\|_{C^{n}}= & \sup _{(r, \theta) \in[0,1] \times \mathbf{R}}\|H(r, \theta)-G(r, \theta)\| \\
& +\sup _{\substack{(r, \theta) \in[0,1] \times \mathbf{R} \\
1 \leq i \leq n}}\left\|D^{i}(H)(r, \theta)-D^{i}(G)(r, \theta)\right\|
\end{aligned}
$$

where $\|\cdot\|$ denotes the usual vector and matrix norms, and $H, G$ are lifts of $h, g$ respectively satisfying $H\left(\frac{1}{2}, 0\right), G\left(\frac{1}{2}, 0\right) \in[0,1] \times[0,2 \pi)$.

Remark. From now on, when we use the coordinates induced by $\eta_{1}$ and $\eta$ on $\mathrm{T}$ and $\boldsymbol{A}$ respectively we will not distinguish between the maps $h: \mathbb{T} \rightarrow \mathbb{T}, h: A \rightarrow A$ and their lifts to $\mathbb{R}$ and $[0,1] \times \mathbb{R}$ respectively. For example, in the above, if $h, g: A \rightarrow A$ are continuous degree one maps then we will write

$$
\|h, g\|_{C^{0}}=\sup _{\theta}\|h(\theta)-g(\theta)\| .
$$

Definition. If $f: \mathbb{J} \rightarrow \mathbb{T}$ (or $f: \mathbb{R} \rightarrow \mathbb{R}$ ) and $\theta_{1}<\theta_{2}$ are such that $f\left(\theta_{1}\right)=\theta_{2}$ then $\left[\theta_{1}, \theta_{2}\right]$ is called a fundamental interval of $f$. 
We will use the following terminology from [4]:

Definition. If $X$ is a metric space, $C$ a compact subset of $X$ and $f: X \rightarrow X$ a continuous map then we call $C$ an attractor block for $f$ if

$$
f(C) \subseteq \text { interior } C \text {. }
$$

The set

$$
\bigcap_{i=1}^{\infty} f^{i}(C) \subseteq C,
$$

which is the largest invariant set in $C$, is called the attractor for the attractor block $C$.

Notation. If $U$ is a subset of $\mathbb{R}^{n}, f: U \rightarrow U$ a $C^{\infty}$ diffeomorphism and $x \in$ interior $U$ such that $f(x)=x$ then we let

$$
\begin{array}{ll}
W^{s}(x, f)=\left\{z \in U: f^{i}(z) \rightarrow x\right. & \text { as } i \rightarrow \infty\} \\
W^{u}(x, f)=\left\{z \in U: f^{i}(z) \rightarrow x\right. & \text { as } i \rightarrow-\infty\} .
\end{array}
$$

If $D f(x)$ has eigenvalues with modulus one then we call the invariant manifold corresponding to the eigenvalues with modulus less than one the strong-stable manifold and we denote it by $W^{s s}(x, f)$.

We will let $T A$ denote the tangent bundle to $A$, and $T_{z} A$ the tangent space to $A$ at $z$. The $(r, \theta)$ coordinates on $A$ induce coordinates on $T_{z} A$. We let $\partial / \partial r$ denote the unit vector in the $r$-direction and $\partial / \partial \theta$ the unit vector in the $\theta$-direction.

We will find it convenient to deal with the projective (or line) bundle over $A$. At each point $z \in A$, each line through the origin of $T_{z} A$ intersects the circle

$$
\left.\left\{x \frac{\partial}{\partial r}+y \frac{\partial}{\partial \theta}:\left(x-\frac{1}{2}\right)^{2}+y^{2}=\frac{1}{4}\right)\right\}
$$

at the origin and at one other point (except the $y$ axis, see figure 1). Hence we can identify the set of lines through the origin with the circle

$$
\left\{x \frac{\partial}{\partial r}+y \frac{\partial}{\partial \theta}:\left(x-\frac{1}{2}\right)^{2}+y^{2}=\frac{1}{4}\right\} \text {. }
$$

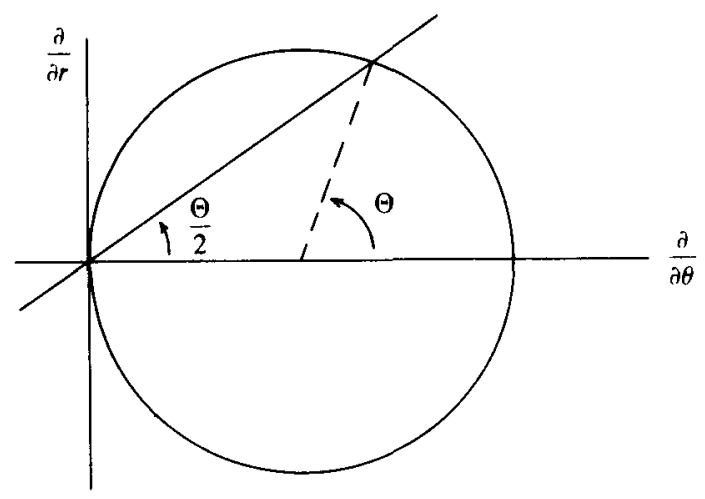

FIGURE 1 
In particular, we can put coordinates on this set of lines by taking twice the arctangent of the slope. This is precisely the angle of intersection between the ray $\left\{x(\partial / \partial r): x \geq \frac{1}{2}\right\}$ and the segment connecting $\left(\frac{1}{2} \partial / \partial r\right)$ with the point of intersection away from the origin (or the origin for the $y$-axis). Hence, letting $P$ denote the projective bundle over $A$, then $P=A \times \mathbb{T}$ and we will denote the coordinates just described by $(r, \theta, \Theta)$. Again, when using these coordinates we will let the context determine whether we are speaking of a map to $P$ or its covering space $[0,1] \times \mathbb{R} \times \mathbb{R}$.

If $f: A \rightarrow A$ is a diffeomorphism then $D f(z): T_{z} A \rightarrow T_{f(z)} A$ preserves the set of lines through the origin and hence $f$ induces a map from $P$ to itself which we will denote by $f_{*}$. Similarly, if $\gamma: \mathbb{T} \rightarrow A$, then the derivative of $\gamma$ induces a map $\gamma_{*}: \mathbb{T} \rightarrow P$.

We will let $Q$ denote the projective bundle over $\mathbb{R}^{2} \sim\{(0,0)\}$. Note that polar coordinates $(r, \theta)$ on $\mathbb{R}^{2} \sim\{(0,0)\}$ induce coordinates on $Q$ exactly as above and we will also use $(r, \theta, \Theta)$ to denote these coordinates.

\section{Review of Hopf bifurcation}

In this section we review the Hopf bifurcation theorem for diffeomorphisms of the plane following the proof of Ruelle and Takens [20]. By making some of the required estimates more precise we will obtain information about the set of parameter values at which particular rotation numbers are assumed on the invariant circle.

For $\mu \in \mathbb{C}$, let $f_{\mu}: \mathbb{R}^{2} \rightarrow \mathbb{R}^{2}$ be as in the introduction, that is, it satisfies

(a) $f_{\mu}$ is jointly $C^{\infty}$ on $\mathbb{C}$ and $\mathbb{R}^{2}$;

(b) for all $\mu \in \mathbb{C}, f_{\mu}(0,0)=(0,0)$;

(c) for all $\mu \in \mathbb{C}$, the eigenvalues of $D f_{\mu}(0,0)$ are $\mu$ and $\bar{\mu}$.

Since we are following Ruelle and Takens [20], we begin by fixing $\zeta_{1}>0$, small, and let

$$
\overrightarrow{\mathbb{C}}=\left\{\mu \in \mathbb{C}:\left|\mu^{n}-1\right| \geq \zeta_{1}, \quad n=1,2,3,4,5\right\} .
$$

From now on we assume $\mu \in \overline{\mathbb{C}}$, avoiding points of 'low order resonance'. The possible behaviour near these parameter values has been discussed in [1]. In particular, the Hopf bifurcation theorem itself is valid about $\mu$ such that $\mu^{5}=1$.

If we write $\mu=s \exp (i \phi)$ then by a $\mu$-dependent change of coordinates we may write $f_{\mu}$ in the polar coordinate 'normal form' as

$$
f_{\mu}(r, \theta)=\left(s r-f_{1}(\mu) r^{3}, \theta+\phi+f_{2}(\mu) r^{2}\right)+\left(\mathcal{O}\left(r^{5}\right), O\left(r^{4}\right)\right),
$$

where $f_{1}, f_{2}: \overline{\mathbb{C}} \rightarrow \mathbb{R}$ are $C^{\infty}$ functions. We assume there exists $\zeta_{2}>0$ such that $f_{1}(\mu)>\zeta_{2}$ for all $\mu \in \overline{\mathbb{C}}$.

Let

$$
\tilde{f}_{\mu}(r, \theta)=\left(s r-f_{1}(\mu) r^{3}, \theta+\phi+f_{2}(\mu) r^{2}\right) .
$$

When $s>1$ the circle

$$
r=\sqrt{\frac{s-1}{f_{1}(\mu)}}
$$

is invariant under $\tilde{f}_{\mu}$. 
LEMMA 1. There exists $\delta_{1}>0$ such that for $\mu=s \exp (i \phi) \in \overline{\mathbb{C}}$ with $1<s<1+\delta_{1}$ the set

$$
U_{\mu}=\left\{(r, \theta): \sqrt{\frac{2(s-1)}{3 f_{1}(\mu)}} \leq r \leq \sqrt{\frac{2(s-1)}{f_{1}(\mu)}}\right\}
$$

is an attractor block for $f_{\mu}$.

Next we point out that there is a corresponding attractor block for $\left(f_{\mu}\right)_{*}$, the lift of $f_{\mu}$ to the projective bundle over the annulus $U_{\mu}$.

LEMMA 2. There exists $\delta_{3}>0$ with $\delta_{3} \leq \delta_{1}$ such that if $\mu=s \exp (i \phi) \in \overline{\mathbb{C}}$ with $1<s<1+\delta_{3}$ then the sets

$$
\begin{aligned}
W_{\mu} & =\{(a, b):|a|=(s-1)|b|\} \\
V_{\mu} & =\{(a, b):|a| \leq(s-1)|b|\}
\end{aligned}
$$

satisfy

$$
D f_{\mu}(r, \theta)\left(a \frac{\partial}{\partial r}+b \frac{\partial}{\partial \theta}\right) \in V_{\mu}
$$

whenever $(a, b) \in W_{\mu}$ and $(r, \theta) \in U_{\mu}$. (See figure 2.)

The proofs of lemmas 1 and 2 are elementary and are essentially contained in the proof of [20, theorem 7.2 ], so we omit them.

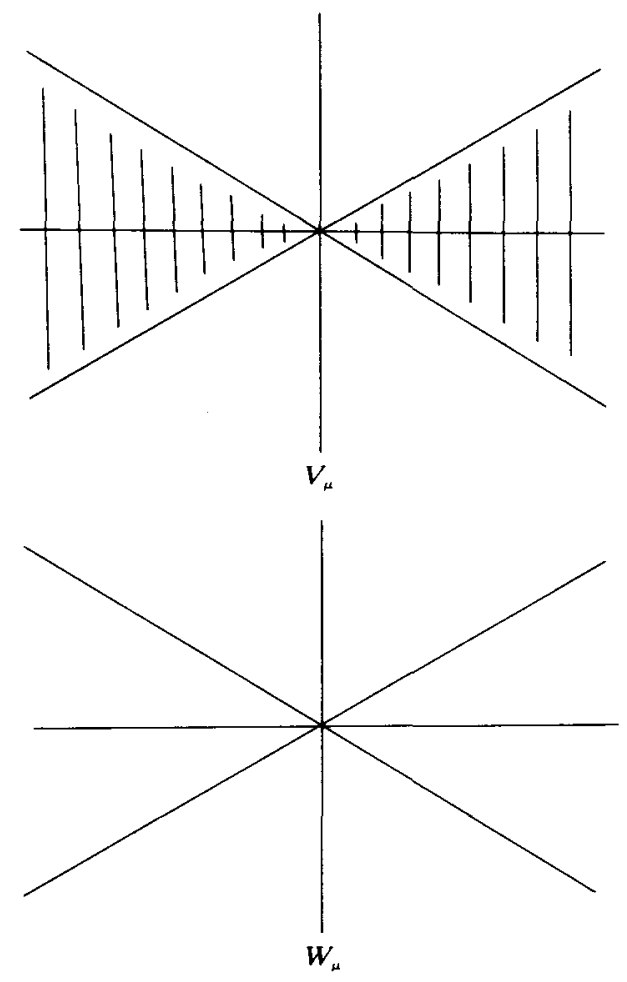

FiguRE 2 
Now by the linearity of $D f_{\mu}$ it follows that for $\mu \in \overline{\mathbb{C}}$ and $1<|\mu|<1+\delta_{3}$ we have

$$
D f_{\mu}(r, \theta) V_{\mu} \subseteq V_{\mu}
$$

whenever $(r, \theta) \in U_{\mu}$. In fact, the above suffices to show that there exists a constant $c_{3}>0$ such that the set

$$
\tilde{V}_{\mu}=\left\{(r, \theta, \Theta) \in Q:(r, \theta) \in U_{\mu},-c_{3}(s-1)<\Theta<c_{3}(s-1)\right\}
$$

is an attractor block for $\left(f_{\mu}\right)_{*}$ when $\mu$ is as above.

Using inequalities such as these lemmas, Ruelle and Takens [20] proceed to show that when $|\mu|$ is sufficiently close to one there is a Lipschitz invariant circle for $f_{\mu}$. By using the invariant manifold theorems of [14] they obtain some differentiability properties for this circle. Specifically they show the following:

Hopf Bifurcation Theorem. For $f_{\mu}$ as above and for $n$ a positive integer there exists $\alpha_{n}>0$ such that if $\mu \in \overline{\mathbb{C}}$ and $1<|\mu|<1+\alpha_{n}$, then there exists a $C^{n}$ attracting invariant circle which surrounds the origin and whose domain of attraction includes every point in its interior except the origin.

Having shown that $f_{\mu}$ has an invariant circle we may ask for which parameter values $f_{\mu}$ restricted to its invariant circle has a given rotation number. Arnol'd [1] has shown that given a rational $p / q \in[0,1]$ with $q>4$ the set $\mu \in \overline{\mathbb{C}}$ for which the rotation number of $f_{\mu}$ on its invariant circle is $p / q$ is contained in (and is generically) a horn shaped region with tip at $\exp (2 \pi i p / q)$ (see figure 3$)$. He also gives a formula

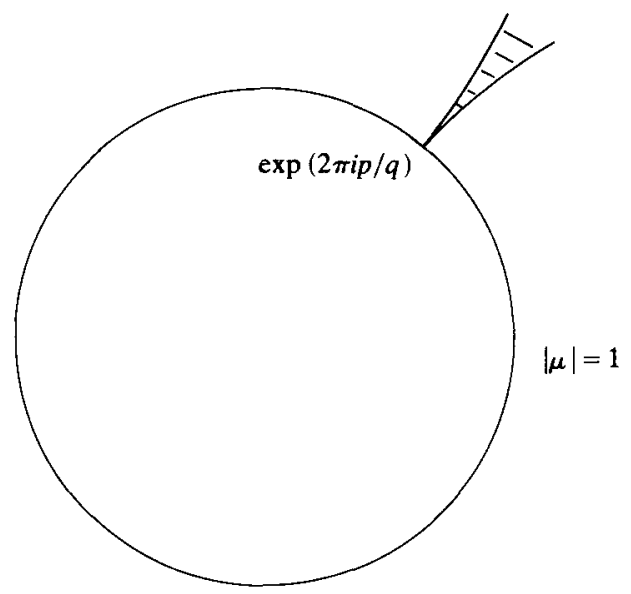

FIGURE 3. Shaded region contains all values of $\mu$ for which $f_{\mu}$ has a point with rotation number $p / q$.

for the degrae of contact between the edges of the horn. We can obtain information about the set of parameter values where an irrational rotation number is assumed by considering more carefully how the invariant circle changes with $\mu \in \overline{\mathbb{C}}$.

Fix $\delta_{4}>0$ such that $\alpha_{2} \geq \delta_{4}$ and $\delta_{3} \geq \delta_{4}$ and such that when $\mu \in \overline{\mathbb{C}}, 1<|\mu|<1+\delta_{4}$; the invariant circle of $f_{\mu}$ given by the Hopf bifurcation theorem may be represented 
as the graph of a function $g_{\mu}:[0,2 \pi) \rightarrow(0, \infty)$, i.e. the invariant circle for $f_{\mu}$ is

$$
\left\{(r, \theta): r=g_{\mu}(\theta)\right\} \text {, }
$$

where $g_{\mu}$ is $C^{2}$. The rest of this section will be devoted to the discussion and proof of the following theorem.

THEOREM 1. Fix an open set $E \subseteq[0,1]$ such that

$$
E \subseteq\{\alpha \in[0,1]: \exp (2 \pi i \alpha) \in \overline{\mathbb{C}}\} .
$$

Then there exist constants $L$ and $\delta_{5}>0$ such that $\delta_{5} \leq \delta_{4}$ and for any irrational $\beta \in E$ there is a Lipschitz curve

$$
\gamma_{\beta}:\left[1,1+\delta_{5}\right) \rightarrow[0,2 \pi)
$$

with Lipschitz constant $L$ such that, if $\mu \in \overline{\mathbb{C}}$ and $1<|\mu|<1+\delta_{5}$, then $f_{\mu}$ restricted to its invariant circle $\left\{\left(g_{\mu}(\theta), \theta\right)\right\}$ has rotation number $\beta$ if and only if $\mu=s \exp \left(i \gamma_{\beta}(s)\right)$ for some s.

Remarks. (1) The fact that the regions of the $\mu$ plane where a particular rotation number is assumed extend a uniform distance from the $|\mu|=1$ circle away from low order resonance was shown (in the setting of flows on the torus) in [3].

(2) The edges of the regions where a given rational rotation number is assumed are given by the implicit function theorem and hence will be at least piecewise $C^{\infty}$. The proof below shows that the edges of these horns are Lipschitz where the Lipschitz constant is independent of the rational $p / q \in E$ (see figure 4).

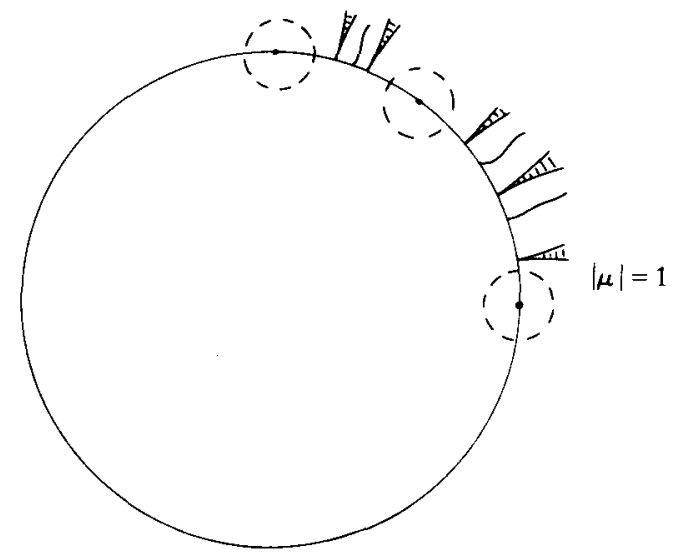

FIgURE 4. The invariant circle has rational rotation number under $f_{\mu}$ for parameter values in the shaded regions. The invariant circle has irrational rotation number for values of $\mu$ on Lipschitz arcs between the shaded regions.

Define, for $\mu=s \exp (i \phi) \in \overline{\mathbb{C}}$ and $1<s<1+\delta_{4}$

$$
\begin{aligned}
& h(\cdot, \phi, s): \mathbb{T} \rightarrow \mathbb{T} \\
& h(\cdot, \phi, s): \theta \rightarrow \lambda_{2} \circ f_{s \exp (i \phi)}\left(g_{s \exp (i \phi)}(\theta), \theta\right) .
\end{aligned}
$$

This gives a two parameter family of homeomorphisms of the circle. If we can 
show that $h$ is Lipschitz in $\phi$ and $s$ then theorem 1 will follow easily. To obtain information on how $h$ varies with $\phi$ and $s$ we must establish estimates of how $g_{\mu}$ varies with $\mu$. In lemma 3 we fix a narrower attractor block containing $g_{\mu}$ than was done in lemma 1. In lemma 4 we give an estimate of how $g_{\mu}(\theta)$ varies with $\mu$ particularly near $|\mu|=1$. This is accomplished by changing coordinates for a particular $\mu_{0}$ so that in the new coordinates the invariant circle has centre at the origin and a constant radius. Then the implicit function theorem can be applied to bound

$$
g_{\mu_{0}+\Delta_{\mu}}(\theta)-g_{\mu_{0}}(\theta)
$$

in terms of $\left|\Delta_{\mu}\right|$. Finally, in lemma 5 we apply the estimates of lemma 4 to obtain estimates on $h(\cdot, \phi, s)$ from which the theorem follows.

LemMA 3. There exist constants $\delta_{6}$ and $c_{4}>0$ such that $\delta_{6} \leq \delta_{4}$ and for $\mu \in \overline{\mathbb{C}}$, $1<|\mu|<1+\delta_{6}$ and $\mu=s \exp (i \phi)$ we have

$$
\left|g_{\mu}(\theta)-\sqrt{\frac{s-1}{f_{1}(\mu)}}\right|<c_{4}(s-1)^{\frac{3}{2}}
$$

Proof. From equations (3.1) and (3.2) we see that there exists a constant $c_{5}>0$ such that for $\mu \in \overline{\mathbb{C}}, 1<|\mu|<1+\delta_{4}$ we have

$$
\left|\frac{\partial\left(\lambda_{1} \circ f_{\mu}\right)}{\partial r}(r, \theta)-\frac{\partial\left(\lambda_{1} \circ \tilde{f}_{\mu}\right)}{\partial r}(r, \theta)\right|<c_{5} r^{4} .
$$

Hence we may fix $\delta_{6}>0$ so small that $\delta_{6} \leq \delta_{4}$ and for $\mu \in \overline{\mathbb{C}}, 1<|\mu|<1+\delta_{6}$ and $(r, \theta) \in U_{\mu}$ we have

So when

$$
0<\frac{\partial\left(\lambda_{1} \circ f_{\mu}\right)}{\partial r}(r, \theta)<1-\frac{s-1}{2}=\frac{3-s}{2}<1
$$

we have

$$
\sqrt{\frac{s-1}{f_{1}(\mu)}}<r \leq \sqrt{\frac{2(s-1)}{f_{1}(\mu)}}
$$

$$
\left(\lambda_{1} \circ f_{\mu}\right)(r, \theta) \leq\left(\lambda_{1} \circ f_{\mu}\right)\left(\sqrt{\frac{s-1}{f_{1}(\mu)}}, \theta\right)+\frac{3-s}{2}\left(r-\sqrt{\frac{s-1}{f_{1}(\mu)}}\right) .
$$

If we fix $c_{6}$ so that $\left|\lambda_{1} \circ f_{\mu}(r, \theta)-\lambda_{1} \circ \tilde{f}_{\mu}(r, \theta)\right|<c_{6} r^{5}$ for all $\mu$ as above then we see that

Hence if

$$
\lambda_{1} \circ f_{\mu}(r, \theta) \leq \lambda_{1} \circ \tilde{f}_{\mu}\left(\sqrt{\frac{s-1}{f_{1}(\mu)}}, \theta\right)+c_{6}\left(\sqrt{\frac{s-1}{f_{1}(\mu)}}\right)^{5}+\frac{3-s}{2}\left(r-\sqrt{\frac{s-1}{f_{1}(\mu)}}\right) .
$$

$$
\left(r-\sqrt{\frac{s-1}{f_{1}(\mu)}}\right)-\frac{3-s}{2}\left(r-\sqrt{\frac{s-1}{f_{1}(\mu)}}\right)>c_{6}\left(\sqrt{\frac{s-1}{f_{1}(\mu)}}\right)^{5}
$$

then

$$
\left(\lambda_{1} \circ f_{\mu}\right)(r, \theta)<r
$$

But $(*)$ holds if and only if

$$
r-\sqrt{\frac{s-1}{f_{1}(\mu)}} \geq c_{6} \frac{2(s-1)^{\frac{3}{2}}}{f_{1}(\mu)^{\frac{5}{2}}} .
$$


By a similar argument for $r<\sqrt{(s-1) / f_{1}(\mu)}$ we see that there exists a constant $c_{4}>0$ such that

$$
\tilde{U}_{\mu}=\left\{(r, \theta): \sqrt{\frac{s-1}{f_{1}(\mu)}}-c_{4}(s-1)^{\frac{3}{2}} \leq r \leq \sqrt{\frac{s-1}{f_{1}(\mu)}}+c_{4}(s-1)^{\frac{3}{2}}\right\}
$$

is an attractor block for $f_{\mu}$ whenever $\mu \in \overline{\mathbb{C}}, 1<|\mu|<1+\delta_{6}$ and the proof of the lemma is complete.

LEMMA 4. There exist constants $\delta_{7}$ and $c_{7}>0$ such that $\delta_{7} \leq \delta_{6}$ and if $\mu$ and $\nu \in \overline{\mathbb{C}}$, $1<|\mu|<1+\delta_{7}, 1<|\nu|<1+\delta_{7}$ and $|\mu-\nu|$ is sufficiently small, then

$$
\left|g_{\nu}(\theta)-g_{\mu}(\theta)\right|<c_{7}\left[|\Delta \phi|+|\Delta s| /(|\mu|-1)^{\frac{1}{2}}\right],
$$

where $(\Delta s, \Delta \phi)$ is the difference of $\mu$ and $\nu$ in polar coordinates.

Proof. Fix $\mu_{0} \in \overline{\mathbb{C}}$ with $1<\left|\mu_{0}\right|<1+\delta_{6}$ and fix $\kappa_{1}>0$ so small that when $\nu \in \mathbb{C}$, $\left|\mu_{0}-\nu\right|<\kappa_{1}$ implies $\nu \in \overline{\mathbb{C}}$,

$$
1<|\nu|<1+\delta_{6} \text { and } \frac{9}{10}<\frac{\left|\mu_{0}\right|-1}{|\nu|-1}<\frac{10}{9} .
$$

With $s_{0} \exp \left(i \phi_{0}\right)=\mu_{0}$, define

$$
\begin{gathered}
\Phi: R^{2} \sim\left\{(r, \theta): r<c_{4}\left(s_{0}-1\right)^{\frac{3}{2}}\right\} \rightarrow \mathbb{R}^{2} \\
\quad:(r, \theta) \rightarrow\left(r-g_{\mu_{0}}(\theta)+\sqrt{\frac{s_{0}-1}{f_{1}\left(\mu_{0}\right)}}, \theta\right) .
\end{gathered}
$$

Then

$$
\Phi^{-1}(r, \theta)=\left(r+g_{\mu_{0}}(\theta)-\sqrt{\frac{s_{0}-1}{f_{1}\left(\mu_{0}\right)}}, \theta\right)
$$

whenever it is defined. Let

$$
k_{\mu}=\Phi \circ f_{\mu} \circ \Phi^{-1}
$$

Noting that

$$
\Phi^{-1}\left(\left\{\left(\sqrt{\frac{s_{0}-1}{f_{1}\left(\mu_{0}\right)}}, \theta\right)\right\}\right)=\left\{\left(g_{\mu_{0}}(\theta), \theta\right)\right\}
$$

we see that the circle $r=\sqrt{\left(s_{0}-1\right) / f_{1}\left(\mu_{0}\right)}$ is invariant under $k_{\mu_{0}}$. Letting

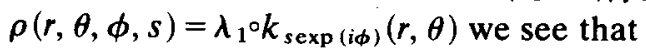

$$
\rho\left(\sqrt{\frac{s_{0}-1}{f_{1}\left(\mu_{0}\right)}}, \theta, \phi_{0}, s_{0}\right)=\sqrt{\frac{s_{0}-1}{f_{1}\left(\mu_{0}\right)}} .
$$

Moreover, since for $\mu=s \exp (i \phi)$

$$
\begin{aligned}
\rho(r, \theta, \phi, s)= & \sqrt{\frac{s_{0}-1}{f_{1}\left(\mu_{0}\right)}}-g_{\mu_{0}}\left(\lambda_{2} \circ f_{\mu}\left(\Phi^{-1}(r, \theta)\right)\right)+\lambda_{1} \circ f_{\mu}\left(\Phi^{-1}(r, \theta)\right) \\
= & \sqrt{\frac{s_{0}-1}{f_{1}\left(\mu_{0}\right)}}-g_{\mu_{0}}\left(\lambda_{2} \circ f_{\mu}\left(\Phi^{-1}(r, \theta)\right)\right) \\
& +s\left(r+g_{\mu_{0}}(\theta)-\sqrt{\frac{s_{0}-1}{f_{1}\left(\mu_{0}\right)}}\right)
\end{aligned}
$$




$$
\begin{aligned}
& -\left(r+g_{\mu_{0}}(\theta)-\sqrt{\frac{s_{0}-1}{f_{1}\left(\mu_{0}\right)}}\right)^{3} f_{1}(\mu) \\
& +\left(r+g_{\mu_{0}}(\theta)-\sqrt{\frac{s_{0}-1}{f_{1}\left(\mu_{0}\right)}}\right)^{5} \rho_{1}(r, \theta, \phi, s),
\end{aligned}
$$

where $\rho_{1}$ is a smooth function, we have

$$
\begin{aligned}
\frac{\partial \rho}{\partial r}(r, \theta, \phi, s)= & -g_{\mu_{0}}^{\prime}\left(\lambda_{2} \circ f_{\mu}\left(r+g_{\mu_{0}}(\theta)-\sqrt{\frac{s_{0}-1}{f_{1}\left(\mu_{0}\right)}}, \theta\right)\right) \\
& \left.\cdot \frac{\partial}{\partial r}\left(\lambda_{2} \circ f_{\mu}\left(r+g_{\mu_{0}}(\theta)-\sqrt{\frac{s_{0}-1}{f_{1}\left(\mu_{0}\right)}}, \theta\right)\right)\right) \\
& +s-3\left(r+g_{\mu_{0}}(\theta)-\sqrt{\frac{s_{0}-1}{f_{1}\left(\mu_{0}\right)}}\right)^{2} f_{1}(\mu) \\
& +O\left(\left(r+g_{\mu_{0}}(\theta)-\sqrt{\frac{s_{0}-1}{f_{1}\left(\mu_{0}\right)}}\right)^{4}\right) .
\end{aligned}
$$

By lemma 2 we may assume $\left|g_{\mu_{0}}^{\prime}(\theta)\right|<\left(s_{0}-1\right)$ and if we choose $s$ and $\phi$ so that $\left|\mu_{0}-s \exp (i \phi)\right|<\kappa_{1}$ then

$$
\frac{9}{10}<\frac{s_{0}-1}{s-1}<\frac{10}{9}
$$

Hence there exists a constant $c_{8}>0$ independent of $\mu$ such that for $(r, \theta) \in U_{\mu_{0}}$ we have

$$
\begin{aligned}
\frac{\partial \rho}{\partial r}(r, \theta, \phi, s)= & \left(s-3 r^{2} f_{1}(\mu)\right) \\
& -g_{\mu_{0}}^{\prime}\left(\lambda_{2} \circ f_{\mu}\left(r+g_{\mu_{0}}(\theta)-\sqrt{\frac{s_{0}-1}{f_{1}\left(\mu_{0}\right)}}, \theta\right)\right) \\
& \cdot \frac{\partial}{\partial r}\left(\left(\lambda_{2} \circ f_{\mu}\right)\left(r+g_{\mu_{0}}(\theta)-\sqrt{\frac{s_{0}-1}{f_{1}\left(\mu_{0}\right)}}, \theta\right)\right) \\
& -6 r\left(g_{\mu_{0}}(\theta)-\sqrt{\frac{s_{0}-1}{f_{1}\left(\mu_{0}\right)}}\right) f_{1}(\mu) \\
& -3\left(g_{\mu_{0}}(\theta)-\sqrt{\frac{s_{0}-1}{f_{1}\left(\mu_{0}\right)}}\right)^{2} f_{1}(\mu) \\
& +O\left(\left(r+g_{\mu_{0}}(\theta)-\sqrt{\frac{s_{0}-1}{f_{1}\left(\mu_{0}\right)}}\right)\right) \\
\leq & 1-\frac{8}{9}\left(s_{0}-1\right) f_{1}(\mu) / f_{1}\left(\mu_{0}\right)+c_{8}\left(s_{0}-1\right)\left(\sqrt{s_{0}-1}\right) \\
& +c_{8}\left(\sqrt{s_{0}-1}\right)\left(s_{0}-1\right)^{\frac{3}{2}}+c_{8}\left(s_{0}-1\right)^{3}+c_{8}\left(s_{0}-1\right)^{2} .
\end{aligned}
$$

Hence we may choose $\delta_{7}>0$ so small that $\delta_{7} \leq \delta_{6}$ and when $\mu_{0} \in \overline{\mathbb{C}}, 1<\left|\mu_{0}\right|<1+\delta_{7}$ and $s \exp (i \phi) \in \overline{\mathbb{C}}$ satisfies $\left|\mu_{0}-s \exp (i \phi)\right|<\kappa_{1}$, then

$$
0<\frac{\partial \rho}{\partial r}(r, \theta, \phi, s)<1-\frac{s_{0}-1}{2}<1 .
$$


Hence we can apply the implicit function theorem to obtain a map $\sigma(\theta, \phi, s)$ such that

$$
\sigma\left(\theta, \phi_{0}, s_{0}\right)=\sqrt{\frac{s_{0}-1}{f_{1}\left(\mu_{0}\right)}}
$$

and

$$
\rho(\sigma(\theta, \phi, s), \theta, \phi, s)=\sigma(\theta, \phi, s)
$$

whenever $\left|\mu_{0}-s \exp (i \phi)\right|<\kappa_{2}$ for a fixed $\kappa_{2}>0, \kappa_{2} \leq \kappa_{1}$. Moreover

$$
\frac{\partial \sigma}{\partial \phi}=\frac{\partial \rho / \partial \phi}{1-\partial \rho / \partial r} \quad \text { and } \quad \frac{\partial \sigma}{\partial s}=\frac{\partial \rho / \partial s}{1-\partial \rho / \partial r}
$$

Now

$$
\begin{aligned}
\frac{\partial \rho}{\partial s}(r, \theta, \varphi, s)= & -g_{\mu_{0}}^{\prime}\left(\lambda_{2} \circ f_{\mu}\left(\Phi^{-1}(r, \theta)\right)\right) \cdot \frac{\partial}{\partial s}\left(\lambda_{2} \circ f_{\mu}\left(\Phi^{-1}(r, \theta)\right)\right) \\
& +\left(r+g_{\mu_{0}}(\theta)-\sqrt{\frac{s_{0}-1}{f_{1}\left(\mu_{0}\right)}}\right)-\left(r+g_{\mu_{0}}(\theta)-\sqrt{\frac{s_{0}-1}{f_{1}\left(\mu_{0}\right)}}\right)^{3} \frac{\partial f_{1}}{\partial s}(\mu) \\
& +O\left(\left(r+g_{\mu_{0}}(\theta)-\sqrt{\frac{s_{0}-1}{f_{1}\left(\mu_{0}\right)}}\right)^{5}\right) .
\end{aligned}
$$

Hence, as above, when $(r, \theta) \in U_{\mu_{0}}$ there is a constant $c_{9}>0$ such that

$$
\begin{aligned}
\left|\frac{\partial \rho}{\partial s}(r, \theta, \phi, s)\right| \leq & c_{9}\left(s_{0}-1\right)\left(s_{0}-1\right)+c_{9}\left[\sqrt{s_{0}-1}+\left(s_{0}-1\right)^{\frac{3}{2}}\right] \\
& +c_{9}\left[\sqrt{s_{0}-1}+\left(s_{0}-1\right)^{\frac{3}{2}}\right]^{3}+c_{9}\left(s_{0}-1\right)^{\frac{5}{2}} .
\end{aligned}
$$

A similar argument for $\partial \rho / \partial \phi$ shows that, by taking $\delta_{6}$ smaller if necessary, there exist constants $c_{9}$ and $c_{10}$ so that

$$
\begin{aligned}
& \left|\frac{\partial \rho}{\partial s}(r, \theta, \phi, s)\right| \leq c_{9} \sqrt{s_{0}-1}, \\
& \left|\frac{\partial \rho}{\partial \phi}(r, \theta, \phi, s)\right| \leq c_{10}\left(s_{0}-1\right) .
\end{aligned}
$$

So letting $c_{11}=\max \left(2 c_{9}, 2 c_{10}\right)$ we have

$$
\begin{aligned}
& \left|\frac{\partial \sigma}{\partial \phi}\right|<\frac{c_{10}\left(s_{0}-1\right)}{1-\left(1-\left(s_{0}-1\right) / 2\right)} \leq c_{11}, \\
& \left|\frac{\partial \sigma}{\partial s}\right|<\frac{c_{9} \sqrt{s_{0}-1}}{1-\left(1-\left(s_{0}-1\right) / 2\right)} \leq c_{11} / \sqrt{s_{0}-1}
\end{aligned}
$$

Now fix $\kappa_{3}>0$ with $\kappa_{2} \geq \kappa_{3}$ and such that when $\left|\mu-\mu_{0}\right|<\kappa_{3}$, and $(r, \theta) \in U_{\mu_{0}}$ with $s \exp (i \phi)=\mu$ we have

$$
0 \leq \frac{\partial \rho}{\partial r}(r, \theta, \phi, s)<1-\frac{s_{0}-1}{4}<1
$$

and

$$
c_{11}\left(|\Delta \phi|+\frac{|\Delta s|}{\sqrt{s_{0}-1}}\right)<\frac{1}{8} \max \left\{\left|\sqrt{\frac{s_{0}-1}{f_{1}\left(\mu_{0}\right)}}-\sqrt{\frac{2\left(s_{0}-1\right)}{f_{1}\left(\mu_{0}\right)}}\right|,\left|\sqrt{\frac{s_{0}-1}{f_{1}\left(\mu_{0}\right)}}-\sqrt{\frac{2\left(s_{0}-1\right)}{3 f_{1}\left(\mu_{0}\right)}}\right|\right\},
$$


where $\Delta s \exp (i \Delta \phi)=\mu-\mu_{0}$. Then the set

$$
\left\{(r, \theta): \sqrt{\frac{s_{0}-1}{f_{1}\left(\mu_{0}\right)}}-2 c_{11}\left(|\Delta \phi|+\frac{|\Delta s|}{\sqrt{s_{0}-1}}\right) \leq r \leq \sqrt{\frac{s_{0}-1}{f_{1}\left(\mu_{0}\right)}}+2 c_{11}\left(|\Delta \phi|+\frac{|\Delta s|}{\sqrt{s_{0}-1}}\right)\right\}
$$

is an attractor block for $k_{\mu}$ and hence its image under $\Phi^{-1}$ is an attractor block for $f_{\mu}$, i.e., the set

$$
\left\{(r, \theta): g_{\mu_{0}}(\theta)-2 c_{11}\left(|\Delta \phi|+\frac{|\Delta s|}{\sqrt{s_{0}-1}}\right) \leq r \leq g_{\mu_{0}}(\theta)+2 c_{11}\left(|\Delta \phi|+\frac{|\Delta s|}{\sqrt{s_{0}-1}}\right)\right\}
$$

is an attractor block for $f_{\mu}$. Hence

$$
\left|g_{\mu}(\theta)-g_{\mu_{0}}(\theta)\right|<c_{7}\left(|\Delta \phi|+\frac{|\Delta s|}{\sqrt{s_{0}-1}}\right),
$$

where $c_{7}=2 c_{11}$ and the proof of the lemma is complete.

Now define

i.e.

$$
\psi(\theta, \phi, s)=\left(\lambda_{2} \circ f_{\mu}\right)\left(g_{\mu}(\theta), \theta\right)-\theta-\varphi
$$

$$
h(\theta, \varphi, s)=\theta+\phi+\psi(\theta, \phi, s) .
$$

LEMMA 5. There exist constants $\delta_{8}$ and $M>0$ such that $\delta_{8}<\delta_{7}$ and for $\mu \in \overline{\mathbb{C}}$, $1<|\mu|<1+\delta_{8}$ and $\mu=s \exp ($ i $\phi)$ we have

(a) $|\psi(\theta, \phi, s)|<M(s-1)$;

(b) $|\psi(\theta, \phi+\Delta \phi, s)-\psi(\theta, \phi, s)|<\frac{|\Delta \phi|}{2}$;

(c) $|\psi(\theta, \phi, s+\Delta s)-\psi(\theta, \phi, s)|<M|\Delta s|$;

where $\mu_{1}=(s+\Delta s) \exp (i \phi)$ and $\mu_{2}=s \exp [i(\phi+\Delta \phi)]$ satisfy $\mu_{i} \in \overline{\mathbb{C}}, 1<\left|\mu_{i}\right|<1+\delta_{8}$ for $i=1,2$ and $\mu, \mu_{1}, \mu_{2}$ are in the same component of $\left\{\mu \in \overline{\mathbb{C}}: 1<|\mu|<1+\delta_{8}\right\}$.

Proof. Recall from equation (3.1) that we may write

$$
\left(\lambda_{2} \circ f_{\mu}\right)(r, \theta)=\theta+\phi+f_{2}(\mu) r^{2}+O\left(r^{4}\right) .
$$

Now fix $\mu=s \exp (i \phi)$ and $s>1$ sufficiently small that if $\mu_{1}$ and $\mu_{2}$ differ in polar coordinates from $\mu$ by $(\Delta s, 0),(0, \Delta \phi)$ respectively then by lemma 4

$$
\begin{aligned}
\left|g_{\mu_{1}}^{2}(\theta)-g_{\mu}^{2}(\theta)\right| & =\left|g_{\mu_{1}}(\theta)+g_{\mu}(\theta)\right|\left|g_{\mu_{1}}(\theta)-g_{\mu}(\theta)\right| \\
& \leq \bar{c}|\Delta s| \\
\left|g_{\mu_{2}}^{2}(\theta)-g_{\mu}^{2}(\theta)\right| & \leq \bar{c} \sqrt{s-1}|\Delta \phi|
\end{aligned}
$$

where $\bar{c}>0$ is independent of $\mu$ when $1<|\mu|<1+\delta_{7}$. Hence choosing $\delta_{8}>0$ sufficiently small, with $\delta_{8} \leq \delta_{7}$ we obtain for $\mu \in \overrightarrow{\mathbb{C}}, 1<|\mu|<1+\delta_{8}$,

$$
|\psi(\theta, \phi+\Delta \phi, s)-\psi(\theta, \phi, s)|<|\Delta \phi| / 2
$$

and

$$
|\psi(\theta, \phi, s+\Delta s)-\psi(\theta, \phi, s)|<\bar{c}|\Delta s|
$$

when $\Delta \phi$ and $\Delta s$ are sufficiently small, i.e., $\psi$ is pointwise Lipschitz at each point. But since the Lipschitz constants are independent of $\mu$ the function $\psi$ satisfies the above inequalities for any $\mu_{1}$ and $\mu_{2}$ in the same component of $\{\nu \in \overline{\mathbb{C}}$ : $\left.1<|\nu|<1+\delta_{8}\right\}$ as $\mu$. 
Also for some constant $\tilde{c}>0$

$$
\begin{aligned}
\psi(\theta, \phi, s) & =\lambda_{2} \circ f_{\mu}\left(g_{\mu}(\theta), \theta\right)-\theta-\phi \\
& \leq g_{\mu}^{2}(\theta) f_{2}(\mu)+\mathcal{O}\left(g_{\mu}^{4}(\theta)\right) \\
& \leq \tilde{c}(s-1)
\end{aligned}
$$

for $\mu \in\left\{\nu \in \overline{\mathbb{C}}: 1<|\nu|<1+\delta_{8}\right\}$. Taking $M=\max \{\bar{c}, \tilde{c}\}$, the proof of the lemma is complete.

Lemma 6. (Herman, [12].) Suppose $h_{1}, h_{2}: \mathbb{T} \rightarrow \mathbb{T}$ are degree one homeomorphisms with

$$
H_{1}(x)>H_{2}(x) \quad \text { for every } x \in R,
$$

where $H_{i}$ is a lift of $h_{i}$ satisfying

$$
H_{i}(0) \in[0,2 \pi), \quad i=1,2 .
$$

Then if either rot $\left(h_{1}\right)$ or $\operatorname{rot}\left(h_{2}\right)$ is irrational then

$$
\operatorname{rot}\left(h_{1}\right)>\operatorname{rot}\left(h_{2}\right) \text {. }
$$

Proof. See [12, proposition III 4.1.1].

Proof of theorem 1. For $\mu \in\left\{\nu \in \overline{\mathbb{C}}: 1<|\nu|<1+\delta_{8}\right\}$ the rotation number of $f_{\mu}$ restricted to $\left\{\left(g_{\mu}(\theta), \theta\right)\right\}$ is the same as $\operatorname{rot}(h(\cdot, \phi, s))$ where $\mu=s \exp (i \phi)$, so it suffices to consider only the maps $h(\cdot, \phi, s): \mathbb{T} \rightarrow \mathbb{T}$.

Fix an irrational $\beta \in E$ and suppose

$$
\operatorname{rot}\left(h\left(\cdot, \phi_{0}, s_{0}\right)\right)=\beta
$$

where $s_{0} \exp \left(i \phi_{0}\right) \in \overline{\mathbb{C}}$ and $1<s_{0}<1+\delta_{8}$. Suppose $\Delta \phi, \Delta s$ are chosen so that $0 \leq|\Delta s|<|\Delta \phi| / 4 M$. Then

$$
\begin{aligned}
|h(\theta, \phi+\Delta \phi, s+\Delta s)-h(\theta, \phi, s)|= & |\Delta \phi+\psi(\theta, \phi+\Delta \phi, s+\Delta s)-\psi(\theta, \phi, s)| \\
\geq & |\Delta \phi|-|\psi(\theta, \phi+\Delta \phi, s+\Delta s)-\psi(\theta, \phi, s+\Delta s)| \\
& -|\psi(\theta, \phi, s+\Delta s)-\psi(\theta, \phi, s)| \\
\geq & |\Delta \phi|-\frac{|\Delta \phi|}{2}-M|\Delta s| \geq \frac{|\Delta \phi|}{2}-\frac{|\Delta \phi|}{4}>0 .
\end{aligned}
$$

Hence $\operatorname{rot}(h(\cdot, \phi+\Delta \phi, s+\Delta s)) \neq \beta$.

Next we fix $\delta_{5}>0$ so small that $\delta_{5} \leq \delta_{8}$ and for any $\alpha \in E$ and each $s$ with $1<s<1+\delta_{5}$ there exist $\phi_{1}$ and $\phi_{2}$ such that $s \exp \left(i \phi_{1}\right)$ and $s \exp \left(i \phi_{2}\right) \in \overline{\mathbb{C}}$, and $s \exp \left(i \phi_{1}\right), s \exp \left(i \phi_{2}\right)$ and $\exp (2 \pi i \alpha)$ are in the same component of $\{\mu \in \overline{\mathbb{C}}: 1 \leq|\mu|<$ $\left.1+\delta_{5}\right\}$, and

$$
\operatorname{rot}\left(h\left(\cdot, \phi_{1}, s\right)\right)<\alpha<\operatorname{rot}\left(h\left(\cdot, \phi_{2}, s\right)\right) .
$$

This is possible since by lemma $5, \psi \rightarrow 0$ as $s \rightarrow 1$.

Hence, for a fixed irrational $\beta \in E$ and $s$ satisfying $1<|s|<1+\delta_{5}$ there exists precisely one $\phi_{s}$ such that

$$
\operatorname{rot}\left(h\left(\cdot, \phi_{s}, s\right)\right)=\beta .
$$

Defining $\gamma_{\beta}(s)=\phi_{s}$ and letting $\gamma_{\beta}(1)=2 \pi \beta$, the above shows that $\gamma_{\beta}$ is a Lipschitz curve on $\left[1,1+\delta_{5}\right)$ with Lipschitz constant $L=4 M$ which completes the proof of the theorem. 


\section{Smoothness}

In this section we consider the smoothness of attracting invariant circles of maps of the annulus, particularly when the rotation number is irrational. The tools we use are the invariant manifold theorems of [7] and [14] and the ideas of [22] and [17]. The aim of this section is to gain more information about the smoothness of the invariant circle near Hopf bifurcation and to point out explicitly the effect of the structure of a lift of an annulus map to the projective bundle. The example in $\S 5$ occurs just when this stucture breaks down.

Notation. For $\delta>0$ we let $C_{\delta}, W_{\delta} \subseteq \mathbb{R}^{2}$ denote the sets

$$
\begin{aligned}
C_{\delta} & =\left\{(x, y) \in \mathbb{R}^{2}:|x| \leq \delta|y|\right\}, \\
W_{\delta} & =\mathbb{R}^{2} \sim C_{\delta}=\{(x, y):|x|>\delta|y|\} .
\end{aligned}
$$

(See figure 5.) If $(r, \theta) \in A$ then we let

$$
\begin{aligned}
& C_{\delta}(r, \theta)=\left\{x \frac{\partial}{\partial r}+y \frac{\partial}{\partial \theta} \in T_{(r, \theta)} A:(x, y) \in C_{\delta}\right\}, \\
& W_{\delta}(r, \theta)=\left\{x \frac{\partial}{\partial r}+y \frac{\partial}{\partial \theta} \in T_{(r, \theta)} A:(x, y) \in W_{\delta}\right\} .
\end{aligned}
$$

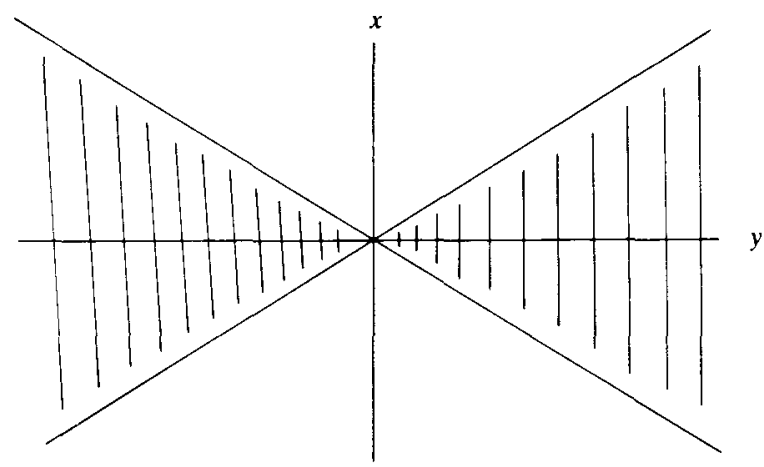

FIGURE 5. The shaded region (including the boundary) is $C_{\delta}$, the complement is $W_{\delta}$.

The $C_{\delta}(r, \theta)$ and $W_{\delta}(r, \theta)$ are called sector bundles over $A$. Let

$$
\tilde{C}_{\delta}(r, \theta)=\left\{(r, \theta, \Theta) \in P \text { : the line represented by }(r, \theta, \Theta) \text { is in } C_{\delta}(r, \theta)\right\} .
$$

Definition. Let $\mathscr{L}$ be the set of $f: A \rightarrow A$ such that:

(1) $f$ is a $C^{\infty}$ diffeomorphism of $A$ into $A$ of degree one,

(2) $A$ is an attractor block for $f$,

(3) there exists $\delta>0$ such that for all $z \in A$

$$
D f(z)\left(C_{\delta}(z)\right) \sim\{(0,0)\} \subseteq \text { interior } C_{\delta}(f(z))
$$

(or $f_{*}\left(\tilde{C}_{\delta}(z)\right) \subseteq$ interior $\left.\tilde{C}_{\delta}(f(z))\right)$,

(4) there exist constants $\alpha$ and $K$ with $0<\alpha<1$ and $K>0$ such that for each $n>0$ and $z \in A$ if $w \in W_{\delta}(z)$ and $D\left(f^{n}\right)(z)(w) \in W_{\delta}\left(f^{n}(z)\right)$ then

$$
\left\|D\left(f^{n}\right)(z)(w)\right\| \leq K \alpha^{n}\|w\| .
$$


THEOREM 2. If $f \in \mathscr{L}$ then the attractor associated with the attractor block $A$ is the graph of a $C^{1}$ map $g: T \rightarrow[0,1]$. If the rotation number of $f$ on the invariant circle is irrational then the circle is a $C^{\infty}$ curve and for each integer $n>0$ there exists $\kappa_{n}>0$ such that if $h: A \rightarrow A$ with $\|h, f\|_{C^{1}<\kappa_{n}}$ then $h$ has a $C^{n}$ invariant circle.

Remark. Referring back to $\S 3$ we see that the above theorem implies that there exists $\delta>0$ such that if $\mu \in \overline{\mathbb{C}}, 1<|\mu|<\delta$ and $f_{\mu}$ has irrational rotation number on its invariant circle then the invariant circle is $C^{\infty}$.

We break the proof of the theorem into several lemmas, first showing in lemma 7 that $f \in \mathscr{L}$ implies $f$ has a Lipschitz invariant circle. In lemma 8 we show that this invariant circle is $C^{1}$ using ideas of [22] and [17]. These first two lemmas also follow from the graph transform techniques of [14], since condition (4) above is the 'hyperbolicity' needed for those techniques. However, we will include a proof of lemma 8 which uses only condition (3) of the definition of $\mathscr{L}$ on the topological structure of the map on the projective bundle over $A$. The example of the next section occurs precisely when this topological stucture breaks down. Finally, after recalling a fact about homeomorphisms of the circle with irrational rotation number, we can apply the invariant manifold theorems of [7] and [14] to complete the proof.

LEMMA 7. If $f \in \mathscr{L}$ then the attractor $U \subseteq A$ associated with the attractor block $A$ is the graph of a Lipschitz function $\mathrm{g}: \mathrm{T} \rightarrow[0,1]$, i.e.,

$$
U=\{(g(\theta), \theta): \theta \in \mathbb{T})\}
$$

Proof. The proof of this lemma is standard and the details are essentially the same as those in [20, proof of theorem 7.2], so we omit it.

LEMMA 8. Suppose $f \in \mathscr{L}$ with invariant circle the graph of the Lipschitz function $g: \mathbb{T} \rightarrow[0,1]$. Then $g$ is actually $a C^{1}$ function.

Proof. Let $P_{g}=\{(r, \theta, \Theta) \in P: r=g(\theta)\}$. Then $f_{*}\left(P_{g}\right)=P_{g}$ and condition (4) of the definition of $\mathscr{L}$ implies that the set

$$
V=\left\{(r, \theta, \Theta) \in \tilde{C}_{\delta}(r, \theta): r=g(\theta)\right\} \subseteq P_{\mathrm{g}}
$$

is an attractor block for $\left.f_{*}\right|_{P_{\mathrm{g}}}$. Let $S \subseteq V$ denote the attractor associated with the attractor block $V$. For each $\theta \in \mathbb{T}$, let

$$
\begin{aligned}
& T_{\theta} g=\left\{(r, \theta, \Theta) \in P_{\mathrm{g}} \text { : there exist sequences }\left\{\theta_{n}\right\}_{n=1}^{\infty},\left\{\theta_{n}^{1}\right\}_{n=1}^{\infty} \text { in } \mathbb{J}\right. \\
& \text { with } \quad \theta_{n} \text { and } \theta_{n}^{1} \rightarrow \theta \quad \text { as } n \rightarrow \infty \\
& \text { and } \left.\tan \left(\frac{\Theta}{2}\right)=\lim _{n \rightarrow \infty} \frac{g\left(\theta_{n}\right)-g\left(\theta_{n}^{1}\right)}{\theta_{n}-\theta_{n}^{1}}\right\} .
\end{aligned}
$$

Then to show that $g$ is $C^{1}$ it suffices to show that $T_{\theta} g$ is a single point for each $\theta \in \mathbb{T}$. But $\bigcup_{\theta \in \mathrm{T}} T_{\theta} g \subseteq V$ and is invariant under $\left.f_{*}\right|_{P_{\mathrm{g}}}$. Hence $\bigcup_{\theta \in \mathrm{T}} T_{\theta} g \subseteq S$ and it suffices to show that for each $\theta \in \mathbb{T}$ the set

$$
S_{\theta}=\{(g(\theta), \theta, \Theta) \in S\}
$$

is a singleton. 
Suppose for some fixed $\theta \in \mathbb{T}$ the set $S_{\theta}$ is not a singleton. Then the sets

$$
I_{n}=\left\{\Theta:(g(\theta), \theta, \Theta) \in V \quad \text { and } \quad f_{*}^{-i}(g(\theta), \theta, \Theta) \in V \quad \text { for } i=1, \ldots, n\right\}
$$

form a nested sequence of intervals with $S_{\theta}=\bigcap_{n=1}^{\infty} I_{n}$. Let $x_{n}$ and $y_{n}$ be the end points of $I_{n}$ and let $v$ and $w$ be the end points of $S_{\theta}$, chosen so that $x_{n} \rightarrow v$ and $y_{n} \rightarrow w$ as $n \rightarrow \infty$, i.e.

$$
I_{n}=\left\{\Theta: y_{n} \leq \Theta \leq x_{n}\right\}, \quad S=\{\Theta: w \leq \Theta \leq v\},
$$

(see figure 6). (We suppress the dependence of $x_{n}, y_{n}, v$ and $w$ on $\theta$.) We define the cross-ratios of $x_{n}, y_{n}, v, w$ to be

$$
C\left(x_{n}, v, w, y_{n}\right)=\frac{\left|\begin{array}{ll}
\cos \left(x_{n} / 2\right) & \cos (v / 2) \\
\sin \left(x_{n} / 2\right) & \sin (v / 2)
\end{array}\right|}{\left|\begin{array}{ll}
\cos \left(x_{n} / 2\right) & \cos \left(y_{n} / 2\right) \\
\sin \left(x_{n} / 2\right) & \sin \left(y_{n} / 2\right)
\end{array}\right|} \frac{\begin{array}{ll}
\cos (w / 2) & \cos \left(y_{n} / 2\right) \\
\sin (w / 2) & \sin \left(y_{n} / 2\right)
\end{array} \mid}{\left|\begin{array}{ll}
\cos (w / 2) & \cos (v / 2) \\
\sin (w / 2) & \sin (v / 2)
\end{array}\right|}
$$

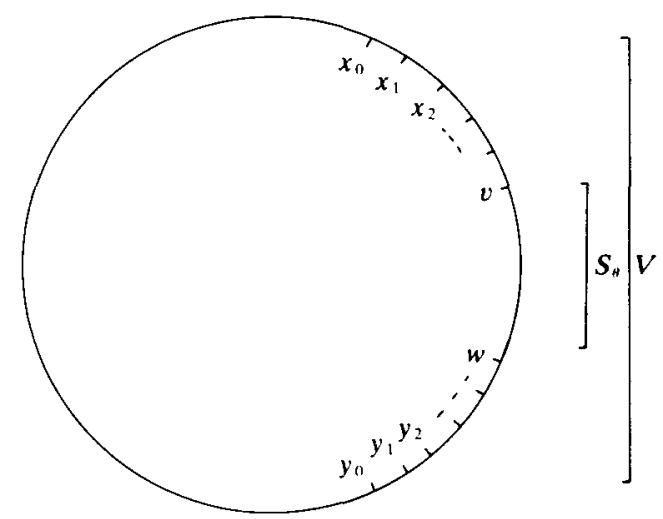

FIGURE 6. The projective space $P$ over $(g(\theta), \theta)$.

Then $C$ is invariant under $f_{*}^{-i}$ for each $i>0$ in the sense that if

$$
\begin{aligned}
& \left(f^{-i}(g(\theta), \theta), \bar{x}_{n}\right)=f_{*}^{-i}\left(g(\theta), \theta, x_{n}\right), \\
& \left(f^{-i}(g(\theta), \theta), \bar{y}_{n}\right)=f_{*}^{-i}\left(g(\theta), \theta, y_{n}\right), \\
& \left(f^{-i}(g(\theta), \theta), \bar{v}\right)=f_{*}^{-i}(g(\theta), \theta, v)
\end{aligned}
$$

and

$$
\left(f^{-i}(g(\theta), \theta), \bar{w}\right)=f_{*}^{-i}(g(\theta), \theta, w)
$$

then

$$
C\left(\bar{x}_{n}, \bar{v}, \bar{w}, \bar{y}_{n}\right)=C\left(x_{n}, v, w, y_{n}\right) .
$$

(See [22].) Now since $x_{n} \rightarrow v$ and $y_{n} \rightarrow w$, for each $\varepsilon>0$ there exists $N$ so that

$$
C\left(x_{n}, v, w, y_{n}\right)<\varepsilon \quad \text { whenever } n \geq N \text {. }
$$

Next we note that if

$$
\left(f^{-n}(g(\theta), \theta), \bar{x}_{n}\right)=f_{*}^{-n}\left(g(\theta), \theta, x_{n}\right)
$$


then $\bar{x}_{n}=x_{0}$ and similarly for $\bar{y}_{n}$. By the compactness of $V$ and continuity of $f_{*}$ there exists $\delta_{1}>0$ such that for every $n$, if $\bar{v}_{n}$ is such that

$$
\left(f^{-n}(g(\theta), \theta), \bar{v}_{n}\right)=f_{*}^{-n}(g(\theta), \theta, v)
$$

and $\bar{w}_{n}$ is such that

$$
\left(f^{-n}(g(\theta), \theta), \bar{w}_{n}\right)=f_{*}^{-n}(g(\theta), \theta, w)
$$

then

$$
\left|x_{0}-\bar{v}_{n}\right|>\delta_{1} \text { and }\left|y_{0}-\bar{w}_{n}\right|>\delta_{1} .
$$

Hence there exists $\delta_{2}>0$ such that

$$
C\left(x_{0}, \bar{v}_{n}, \bar{w}_{n}, y_{0}\right)>\delta_{2}
$$

for every $n$. This contradicts equation (4.1) above which implies that for $n$ sufficiently large

$$
C\left(x_{n}, v, w, y_{n}\right)<\delta_{2} \text {. }
$$

Hence, for each $\theta \in \mathbb{T}, S_{\theta}$ must be a singleton and the proof of the lemma is complete.

Remark. It is interesting to note that in the proof of lemma 8 we did not use conditions (2) or (4) of the definition of $\mathscr{L}$. Hence a diffeomorphism of the annulus which has a Lipschitz invariant circle with the tangent cone of the circle an attractor with attractor block as described in condition (3) of the definition of $\mathscr{L}$, then the circle is actually $C^{1}$.

In order to apply Fenichel's theorem we must consider the derivative of the map restricted to the invariant circle.

Lemma 9. (Denjoy, [6]; Herman, [12].) If $h: \mathbb{T} \rightarrow \mathbb{T}$ is a $C^{1}$ diffeomorphism with irrational rotation number then

$$
\lim _{n \rightarrow \infty}\left[D\left(h^{n}\right)(\theta)\right]^{\frac{1}{n}}=1
$$

for every $\theta \in \mathbb{T}$.

Proof See [12, proposition VI 1.1].

Proof of theorem 2. That a map $f \in \mathscr{L}$ has a $C^{1}$ invariant circle is precisely lemmas 7 and 8 . Then condition (4) of the definition of $\mathscr{L}$ and equation (4.2) applied to the restriction of $f$ to its invariant circle are precisely the hypotheses for Fenichel's theorem, which completes the proof of the theorem.

\section{A Denjoy attractor}

In this section we construct an example proving

THEOREM 3. There exists a $C^{\infty}$ diffeomorphism $h$ from the annulus $A$ into itself such that $\boldsymbol{A}$ is an attractor block for $h$, the attractor associated with $A$ is a Lipschitz circle given by a function $\gamma: \mathbb{T} \rightarrow A$, and $\left.h\right|_{\gamma(\mathrm{T})}$ has no periodic orbits and no dense orbits.

Remark. The set $\gamma(\mathbb{T})$ will be a Lipschitz circle in the sense that it satisfies a 'cone condition' so that in properly chosen coordinates the set $\gamma(\pi)$ is the graph of a Lipschitz function. 
The desired map $h$ will be the limit of a sequence $\left\{h_{n}: A \rightarrow A\right\}_{n=1}^{\infty}$ each $h_{n}$ having a $C^{\infty}$ invariant circle as an attractor, and each $h_{n}$ having a single periodic orbit with periods increasing with $n$. We will construct carefully the first map of the sequence since it serves as a prototype, having the same qualitative features as each of the $h_{n}$ 's.

We begin with a map $f: A \rightarrow A$ which may be defined in $(r, \theta)$-coordinates by

$$
f:(r, \theta) \rightarrow\left(\frac{1}{2}\left(r-\frac{1}{2}\right)+\frac{1}{2}, \tilde{f}(\theta)\right)
$$

where $\tilde{f}: \mathbb{T} \rightarrow \mathbb{T}$ is a degree one, $C^{\infty}$ diffeomorphism of $\mathbb{T}$ which has a single periodic orbit. Let $p_{1} / q_{1}$ be the rotation number of $\tilde{f}$ and let $0<\theta_{1}<\cdots<\theta_{q_{1}}<1$ be the points on the periodic orbit of $\tilde{f}$. Since $\tilde{f}$ has only one periodic orbit it must be a node, i.e.

$$
D\left(\tilde{f}^{q_{1}}\right)\left(\theta_{i}\right)=1, \quad i=1,2, \ldots, q_{1} .
$$

We assume that $D^{2}\left(\tilde{f}^{a_{i}}\right)\left(\theta_{i}\right)>0, i=1, \ldots, q_{1}$. Since the circle $r=\frac{1}{2}$ is an attractor for $f$ with attractor block $A, f$ has only one periodic orbit. This orbit consists of the points $\left(\frac{1}{2}, \theta_{1}\right),\left(\frac{1}{2}, \theta_{2}\right), \ldots,\left(\frac{1}{2}, \theta_{q_{1}}\right)$. The eigenvalues of $D\left(f^{q_{1}}\right)\left(\frac{1}{2}, \theta_{i}\right)$ are 1 in the $\theta$-direction (along the invariant circle), and $\left(\frac{1}{2}\right)^{a_{1}}$ in the $r$-direction. We may characterize the invariant circle as

$$
\bigcup_{i=1}^{q_{1}} W^{u}\left(\left(\frac{1}{2}, \theta_{i}\right), f^{q_{1}}\right),
$$

i.e. the invariant circle is the union of the unstable components of the centre manifolds of the points $\left(\frac{1}{2}, \theta_{i}\right)$ with respect to the map $f^{q_{1}}$.

Next we consider the map $f_{*}: P \rightarrow P$. This map has precisely two periodic orbits which as sets are given by $\left\{\left(\frac{1}{2}, \theta_{i}, 0\right): i=1, \ldots, q_{1}\right\}$ and $\left\{\left(\frac{1}{2}, \theta_{i}, \pi\right), i=1, \ldots, q_{1}\right\}$. The first corresponds to the periodic orbit of $f$ with tangent direction along the invariant circle while the second corresponds to the strong stable direction of the periodic orbit of $f$. The map $f_{*}$ preserves the set $\left\{\left(\frac{1}{2}, \theta, 0\right): \theta \in \mathbb{T}\right\}$ since this is the lift to $P$ of the invariant circle. Also

$$
f_{*}(\{(r, \theta, \pi):(r, \theta) \in A\}) \subseteq\{(r, \theta, \pi):(r, \theta) \in A\} .
$$

This corresponds to the fact that the curves $\theta=$ constant give a smooth, $f$-invariant foliation of $\boldsymbol{A}$ with segments connecting the boundary components of $\boldsymbol{A}$. We note that the strong stable manifolds

$$
W^{s s}\left(\left(\frac{1}{2}, \theta_{i}\right), f^{q_{i}}\right)=\left\{\left(r, \theta_{i}\right): 0 \leq r \leq 1\right\}
$$

are leaves of this foliation for $i=1,2, \ldots, q_{1}$. Finally

$$
\{(r, \theta, \pi):(r, \theta) \in A\}=\bigcup_{i=1}^{a_{1}} W^{s}\left(\left(\frac{1}{2}, \theta_{i}, \pi\right), f_{*}^{q_{1}}\right),
$$

i.e. the invariant surface in the projective bundle corresponding to the invariant foliation is given by the stable components of the centre-stable manifolds of the periodic orbit $\left\{\left(\frac{1}{2}, \theta_{i}, \pi\right): i=1,2, \ldots, q_{1}\right\}$ (see figure 7 ).

We will set $f$ equal to $h_{1}$ the first map of the sequence $\left\{h_{n}: A \rightarrow A\right\}_{n=1}^{\infty}$ which onverges to the desired example. Each of the maps $h_{n}$ will satisfy the description given above for $f$ and we will describe these properties carefully later. 


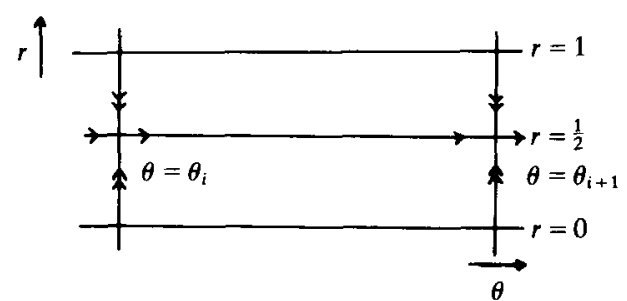

A

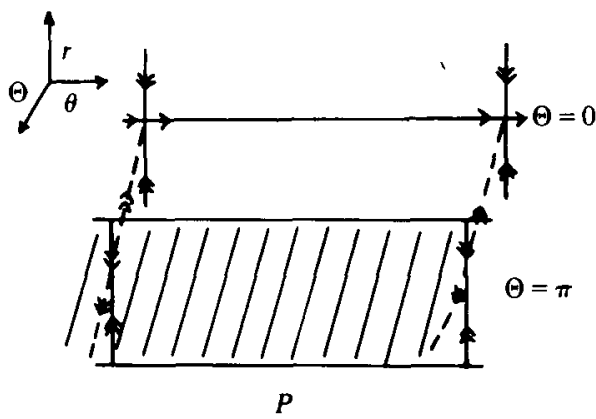

FIGURE 7. Representation of invariant manifolds for $f^{a_{1}}$ in $A$ and $f_{*}^{q_{1}}$ in $P$.

The construction of $h_{2}$ from $f=h_{1}$ (and of $h_{n+1}$ from $h_{n}$ ) takes place in two steps. First we construct a one parameter family of maps $f_{\zeta}, \zeta \in[0,1]$, such that $f_{0}=f$, each $f_{\zeta}$ satisfies the description given above for $f, f$ and $f_{\zeta}$ have precisely the same periodic orbits and $\left(f_{1}\right)_{*}$ has an interval of heteroclinic points between the two periodic orbits in $P$. Loosely we can say that a segment of the invariant circle of $f_{1}$ lies along a leaf of the foliation associated with the strong stable direction of the periodic orbit of $f_{1}$. Hence for $\zeta_{0}$ near one, there will be an interval on the invariant circle whose length decreases at an exponential rate for a large number of iterates. The next step of the construction is to make a small perturbation of the map $f_{\zeta_{0}}$ near the periodic orbit without affecting the invariant foliation, which produces a map with a single periodic orbit with much longer period and which satisfies the conditions described above for $f$. Since the interval on the invariant circle near the leaf of the foliation is small with respect to the fundamental interval which contains it, we can arrange that the new periodic orbit of the perturbed map misses this interval. The result will be the next map in our sequence.

We begin by giving an explicit construction of the one parameter family $f_{\xi}$. This provides motivation for the definitions which follow giving the conditions which each $h_{n}$ must satisfy. Finally we give the induction step described in the paragraph above in lemma 11 .

To construct the one parameter family $f_{6}$ first fix $x_{1}$ and $x_{2} \in\left(\theta_{1}, \theta_{2}\right)$ such that $x_{1}<x_{2}$ and $\tilde{f}^{q_{1}}\left(x_{1}\right)=x_{2}$, i.e. $\left[x_{1}, x_{2}\right]$ is a fundamental interval for $\tilde{f}^{a_{1}}$. Fix $y_{1}<y_{2}$ with $x_{1}<y_{1}<y_{2}<x_{2}$ and

$$
y_{1}+\frac{y_{2}-y_{1}}{2}=x_{1}+\frac{x_{2}-x_{1}}{2} \text {. }
$$

Let $I_{1}=\left\{\frac{1}{2}\right\} \times\left[y_{1}, y_{2}\right]$ and $J_{1}=\left\{\frac{1}{2}\right\} \times\left[x_{1}, x_{2}\right]$. We assume $x_{2}-x_{1} \ll \frac{1}{2}$. Let $\psi: \mathbb{R} \rightarrow \mathbb{R}$ be a $C^{\infty}$ bump function with support contained in $\left(-\left(x_{2}-x_{1}\right) / 2,\left(x_{2}-x_{1}\right) / 2\right), 1 \geq \psi \geq 0$ and $\psi(x)=1$ whenever $|x| \leq\left(y_{2}-y_{1}\right) / 2$. For $\zeta \in[0,1]$ let

$$
\Psi_{\zeta}: \mathbb{R}^{2} \rightarrow \mathbb{R}^{2} ;(a, b) \rightarrow\left(\begin{array}{cc}
\cos \left(\frac{\pi}{2} \zeta \psi(\|(a, b)\|)\right) & \sin \left(\frac{\pi}{2} \zeta \psi(\|(a, b)\|)\right) \\
-\sin \left(\frac{\pi}{2} \zeta \psi(\|(a, b)\|)\right) & \cos \left(\frac{\pi}{2} \zeta \psi(\|(a, b)\|)\right)
\end{array}\right) \cdot\left(\begin{array}{l}
a \\
b
\end{array}\right)
$$


Now we define, for $\zeta \in[0,1]$,

$$
\begin{aligned}
& f_{\zeta}: A \rightarrow A \\
& f_{\zeta}:(r, \theta) \rightarrow f\left(\Psi_{\zeta}\left((r, \theta)-\left(\frac{1}{2}, x_{1}+\frac{x_{2}-x_{1}}{2}\right)\right)+\left(\frac{1}{2}, x_{1}+\frac{x_{1}-x_{2}}{2}\right)\right) .
\end{aligned}
$$

Since $f_{\zeta}$ differs from $f$ only on the strip $[0,1] \times\left(x_{1}, x_{2}\right)$ we see that $f_{\zeta}$ has exactly one periodic orbit made up of the points $\left(\frac{1}{2}, \theta_{1}\right),\left(\frac{1}{2}, \theta_{2}\right), \ldots,\left(\frac{1}{2}, \theta_{q_{1}}\right)$. Moreover, for $\zeta \in[0,1]$ the set

$$
\bigcup_{i=1}^{q_{1}} W^{u}\left(\left(\frac{1}{2}, \theta_{i}\right), f^{q_{1}}\right)
$$

is the invariant circle for $f_{\zeta}$ and when $\zeta<1$ this circle is $C^{\infty}$. Also for $\zeta \in[0,1]$, $\left(f_{\zeta}\right)_{*}$ has precisely two periodic orbits

$$
\left\{\left(\frac{1}{2}, \theta_{i}, 0\right): i=1, \ldots, q_{1}\right\}
$$

and

$$
\left\{\left(\frac{1}{2}, \theta_{i}, \pi\right): i=1, \ldots, q_{1}\right\}
$$

and when $\zeta<1$ the set

$$
\bigcup_{i=1}^{q_{1}} W^{s}\left(\left(\frac{1}{2}, \theta_{i}, \pi\right),\left(f_{\zeta}^{q_{1}}\right)_{*}\right)
$$

is a $C^{\infty},\left(f_{\zeta}\right)_{*}$-invariant surface in $P$ which yields a smooth $f_{\zeta}$-invariant foliation of $A$. When $\zeta=1$, the set

$$
\bigcup_{i=1}^{q_{1}} W^{u}\left(\left(\frac{1}{2}, \theta_{i}\right), f_{1}^{q_{1}}\right)
$$

is a Lipschitz $f_{1}$-invariant circle (i.e. it satisfies a cone condition) and the set

$$
\left\{\left(\frac{1}{2}, \theta, 0\right): y_{1} \leq \theta \leq y_{2}\right\} \subseteq P
$$

is contained in the set

$$
W^{u}\left(\left(\frac{1}{2}, \theta_{1}, 0\right),\left(f_{1}\right)_{*}^{q_{1}}\right) \cap W^{s}\left(\left(\frac{1}{2}, \theta_{2}, \pi\right),\left(f_{1}\right)_{*}^{q_{1}}\right) .
$$

This last statement says that the interval $\left\{\left(\frac{1}{2}, \theta, 0\right): y_{1} \leq \theta \leq y_{2}\right\}$ forms an interval of heteroclinic points between the two periodic orbits of $\left(f_{1}\right)_{*}$. This is a degenerate heteroclinic tangency between the centre manifold of $\left(\frac{1}{2}, \theta_{1}, 0\right)$ and the centre-stable manifold of $\left(\frac{1}{2}, \theta_{2}, \pi\right)$ (see figures 8 and 9). Each of the maps $\left\{h_{n}: A \rightarrow A\right\}_{n=1}^{\infty}$ will

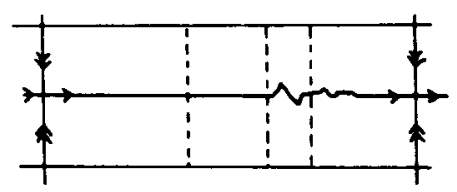

A

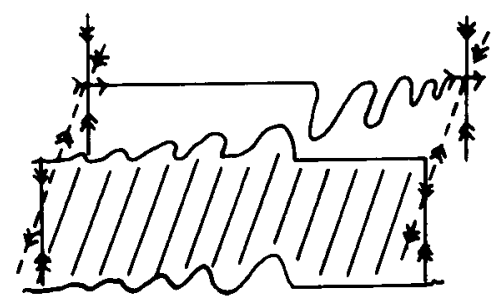

$P$

FIGURE 8. Representation of the invariant manifolds for $\left(f_{2}^{\frac{1}{2}}\right)^{q_{r}}$ and $\left(f_{\frac{1}{2}}\right)_{*}^{q_{1}}$. 


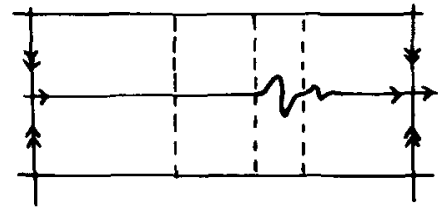

A

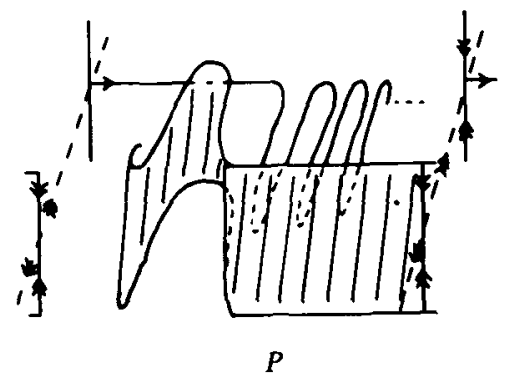

$P$

FIGURE 9. Representation of the invariant manifolds for $\left(f_{1}\right)^{q_{1}}$ and $\left(f_{1}\right)_{*}^{q_{1}}$.

have a one parameter family of maps associated with it which have many of the qualitative features of $f_{\zeta}$ described above with respect to periodic orbits of increasingly large period.

In order to make this precise we make the following tedious technical definitions. Definition. Let $\alpha_{1}$ and $\alpha_{2} \in(0, \pi)$ with $\alpha_{1}<\alpha_{2}$. We say a map $h: A \rightarrow A$ satisfies conditions (1-8) (for $\alpha_{1}, \alpha_{2}$ ) if $h$ satisfies the following conditions:

(1) $h$ is a $C^{\infty}$ diffeomorphism of $A$ into $A$;

(2) $A$ is an attractor block for $h$;

(3) $h$ has exactly one periodic orbit; we let $p / q$ denote the rotation number of this orbit and we let $\left(r_{1}, \theta_{1}\right),\left(r_{2}, \theta_{2}\right), \ldots,\left(r_{q}, \theta_{q}\right)$ denote the points on the orbit;

(4) the eigenvalues of $D\left(h^{a}\right)\left(r_{i}, \theta_{i}\right)$ are 1 and $\alpha$ where $0<\alpha<1$;

(5) the strong stable manifolds $W^{s s}\left(\left(r_{i}, \theta_{i}\right), h^{q}\right)$ are segments connecting the boundary components of $A$;

(6) the set $\bigcup_{i=1}^{q} W^{u}\left(\left(r_{i}, \theta_{i}\right), h^{q}\right)$ is the image of a continuous, injective map $\gamma: \mathbb{T} \rightarrow A$, and $\gamma(\mathbb{T})$ is the attractor for the attractor block $A$;

(7) if $\overline{(r, \theta),\left(r^{\prime}, \theta^{\prime}\right)} \in \gamma(\mathbb{T})$, and $(r, \theta, \Theta) \in P$ represents the line in the tangent space $T_{(r, \theta)} A$ tangent to the segment $(r, \theta),\left(r^{\prime}, \theta^{\prime}\right)$, then $\Theta \notin\left[\alpha_{1}, \alpha_{2}\right]$ (i.e. this is the 'cone condition' for the invariant circle);

(8) for each $i=1, \ldots, q$,

$$
W^{s s}\left(\left(r_{i}, \theta_{i}\right), h^{q}\right) \cap \gamma(\mathbb{T})=\left\{\left(r_{i}, \theta_{i}\right)\right\} .
$$

Definition. We say a map $h: A \rightarrow A$ satisfies conditions (1-12) (for $\alpha_{1}, \alpha_{2}$ as above) if $h$ satisfies conditions (1-8) and $h$ satisfies

(9) $\gamma: \mathbb{T} \rightarrow A$ is a $C^{\infty}$ embedding;

(10) if $\left\{\left(r_{i}, \theta_{i}, \widehat{\Theta}_{i}\right): i=1, \ldots, q\right\} \subseteq P$ are the points on the periodic orbit of $h_{*}$ corresponding to the eigendirection for the eigenvalue $\alpha$, then the set

$$
\bigcup_{i=1}^{q} W^{s}\left(\left(r_{i}, \theta_{i}, \bar{\Theta}_{i}\right), h_{*}^{q}\right)
$$

is the image of a $C^{\infty}$ map $\sigma: A \rightarrow P$ which is a section of the natural projection of $P$ onto $A$;

(11) the vector field given by $\sigma(A)$, (by giving the points of $\sigma(A)$ orientation in a continuous way and unit norm) has solution curves which are segments 
connecting the components of the boundary of $A$, and each such curve intersects $\gamma(\mathbb{T})$ in a single point;

$$
\sigma(A) \cap \gamma_{*}(\mathbb{T})=\varnothing=\sigma(A) \cap\{(s, \theta, 0): s=0,1, \quad \theta \in[0,2 \pi)\}
$$

Remarks. Conditions (10) and (11) can be restated in terms of the existence of a smooth, $h$-invariant foliation. In fact, if $h: A \rightarrow A$ satisfies conditions (1-12) with invariant circle $\gamma: \mathbb{T} \rightarrow A$ then we can let $\Phi$ be the flow associated with the invariant surface in $P$ given by condition (10) as described in (11) by orienting the vectors on $\{(1, \theta): \theta \in[0,2 \pi)\}$ pointing into $A$. Then each solution of $\Phi$ contains exactly one point of $\{1\} \times \pi \subseteq A$ and one point of $\gamma(\pi)$. Hence we can use $\Phi$ to give a coordinate system on $A$ as follows:

If $z \in A$ then let $\Phi(z, \cdot)$ be the solution curve containing $z$ and $\Phi(z, 0)=z$. Let $\tilde{r} \in \mathbb{R}$ be such that $\Phi(z,-\tilde{r}) \in \gamma(\mathbb{T})$ and let $\tilde{\theta} \in \mathbb{T}$ be such that there exists $t \in \mathbb{R}$ with $\Phi(z, t)=(1, \tilde{\theta})$. Then $(\tilde{r}, \tilde{\theta})$ form a smooth coordinate system on $A$. In these coordinates

$$
h:(\tilde{r}, \tilde{\theta}) \rightarrow(k(\tilde{r}, \tilde{\theta}), u(\tilde{\theta}))
$$

i.e. the curves $\tilde{\theta}=$ constant make up the $h$-invariant foliation. Also, $\tilde{r}=0$ is the invariant circle $\gamma(\mathbb{T})$ for $h$, and in these coordinates $u: \mathbb{T} \rightarrow \mathbb{T}$ equals $\left.h\right|_{\gamma(\pi)}$. We will name these coordinates by the diffeomorphism which induces them, e.g. $(\tilde{r}, \tilde{\theta})$ above will be called $h$-coordinates.

We still need one more technical definition which makes precise the notion of a map being $C^{n}$ close to a map with an interval of heteroclinic points in $P$, e.g. $f_{\zeta}$ is close to $f_{1}$ when $\zeta$ is close to 1 . We will need to arrange that the smooth structure (e.g. the invariant foliation) persists until the heteroclinic points appear as is the case for $f_{\zeta}$ described above.

Definition. Suppose $h: A \rightarrow A$ satisfies conditions (1-12) for some $\alpha_{1}, \alpha_{2} \in(0, \pi)$ with invariant circle given by $\gamma: \mathbb{T} \rightarrow A$ and rotation number $p / q$. Suppose $J \subseteq \gamma(\mathbb{T})$ is an interval which is contained in a fundamental interval of $\left.h^{q}\right|_{\gamma(T)}$ and $I \subseteq J$ is an interval which does not contain the end points of $J$. Suppose $U$ is a neighbourhood of $I$ in $A, U \cap \gamma(\mathbb{T})$ is contained in $J$ and $U$ is bounded away from $\gamma(\mathbb{J}) \sim J$. Fix $\delta$ and $n>0$. We say that $h$ is $(\delta, n)$-close-to-connection with respect to $I, J$ and $U$ for $\alpha_{1}, \alpha_{2}$ if there exists a one parameter family $h_{\zeta}: A \rightarrow A$ for $\zeta \in[0,1]$ such that

(a) $h_{0}=h$, and for all $\zeta \in[0,1]$ and all $z \in A \sim U, h_{\zeta}(z)=h(z)$;

(b) for all $\zeta \in[0,1], h_{\zeta}$ satisfies conditions (1-8) for $\alpha_{1}, \alpha_{2}$ and for all $\zeta \in[0,1)$, $h_{\zeta}$ satisfies conditions $(1-12)$ for $\alpha_{1}, \alpha_{2}$;

(c) if $\gamma_{\zeta}: \mathbb{J} \rightarrow A$ is the invariant circle for $h_{\zeta}, \zeta \in[0,1]$, then $\gamma_{\zeta}$ is continuous in $\zeta$ in the $C^{0}$ topology and for each integer $m, h_{\zeta}$ is continuous in the $C^{m}$ topology for $\zeta \in[0,1]$

(d) for $\zeta \in[0,1],\left\|h_{\zeta}, h\right\|_{C^{n}}<\delta$ and $\left\|\gamma_{\zeta}, \gamma_{0}\right\|_{C^{0}}<\delta$;

(e) $J \subseteq \gamma_{\zeta}(\pi)$ for all $\zeta \in[0,1]$ and $U$ is bounded away from $\bigcup_{\zeta \in[0.1]}\left(\gamma_{\zeta}(\pi) \sim J\right)$;

(f) let $\sigma_{\zeta}: A \rightarrow P$ denote the smooth invariant surface in $P$ giving the $h_{\zeta}$-invariant foliation, then there exists an open set $W$ containing $\bigcup_{\zeta \in[0,1]} \gamma_{\zeta}(\mathbb{T})$ such that if 
$\zeta \in[0,1)$ and $(r, \theta, \Theta) \in \sigma_{\zeta}(A)$ with $(r, \theta) \in W$ then $\Theta \notin\left[\alpha_{1}, \alpha_{2}\right]$,

(g) each point of the segment

$$
\left\{(r, \theta, \Theta):(r, \theta) \in I \text { and }(r, \theta, \Theta) \in \gamma_{*}(\mathbb{J})\right\}
$$

is a heteroclinic point for $\left(h_{1}\right)_{*}$ between the two periodic orbits of $\left(h_{1}\right)_{*}$ in $P$.

Remarks and notation. For $h: A \rightarrow A$ as in the definition above we may assume that the points of the periodic orbit $\left(r_{1}, \theta_{1}\right), \ldots,\left(r_{q}, \theta_{q}\right)$ of $h$ are ordered so that for each $z \in I$,

$$
\left(h^{a}\right)^{i}(z) \rightarrow\left(r_{1}, \theta_{1}\right) \quad \text { as } i \rightarrow-\infty
$$

and

$$
\left(h^{q}\right)^{i}(z) \rightarrow\left(r_{2}, \theta_{2}\right) \quad \text { as } i \rightarrow \infty \text {. }
$$

Then let $\left(r_{1}, \theta_{1}, \Theta_{1}\right), \ldots,\left(r_{q}, \theta_{q}, \Theta_{q}\right)$ and $\left(r_{1}, \theta_{1}, \bar{\Theta}_{1}\right), \ldots,\left(r_{q}, \theta_{q}, \bar{\Theta}_{q}\right)$ denote the periodic orbits of $h_{*}$ corresponding to the direction tangent to $\gamma(\mathbb{T})$ and the direction of the strong stable manifold respectively. Since $h_{\zeta}$ equals $h$ near $\left\{\left(r_{i}, \theta_{i}\right): i=1, \ldots, q\right\}$ we see that the periodic orbits of $h_{\zeta}$ and $\left(h_{\zeta}\right)_{*}$ are precisely the same as those of $h$ and $h_{*}$ for any $\zeta \in[0,1]$. By conditions (a) and (e) we see that

$$
\gamma_{\zeta}(\pi) \cap U=\gamma(\pi) \cap U \quad \text { for all } \zeta \in[0,1]
$$

and that the foliation is independent of $\zeta$ on a neighbourhood of $\bigcup_{i=1}^{\infty}\left(h_{\xi}^{q}\right)^{i}(J)$. Finally note that we can restate condition $(g)$ as

$$
\begin{aligned}
& \left\{(r, \theta, \Theta):(r, \theta) \in I \text { and }(r, \theta, \Theta) \in \gamma_{*}(\mathbb{T})\right\} \\
& \quad \subseteq W^{u}\left(\left(r_{1}, \theta_{1}, \Theta_{1}\right),\left(h_{1}\right)_{*}^{q}\right) \cap W^{s}\left(\left(r_{2}, \theta_{2}, \bar{\Theta}_{2}\right),\left(h_{1}\right)_{*}^{q}\right),
\end{aligned}
$$

and note that since $I \subseteq \gamma_{\zeta}(\mathbb{T})$ for each $\zeta \in[0,1]$ we know that $\{(r, \theta, \Theta):(r, \theta) \in I$ and $\left.(r, \theta, \Theta) \in \gamma_{*}(\mathbb{T})\right\}$ is contained in $\left(\gamma_{\zeta}\right)_{*}(\mathbb{\mathbb { T }})$ for each $\zeta \in[0,1)$.

We can now give the induction step of our construction in the following three lemmas. In lemma 10 we take care of a technical detail, showing that a fundamental interval doesn't change much when passing near a point which is almost a fixed point. Lemma 11 is the heart of the construction. Finally in lemma 12 we collect the details of the construction of the desired sequence $\left\{h_{n}: A \rightarrow A\right\}_{n=1}^{\infty}$.

LEMMA 10. Let $v:[-\delta, \delta] \rightarrow \mathbb{R}$ be a $C^{2}$ map such that $v(x) \geq x, \frac{4}{3}>D v(x)>\frac{3}{4}$ and $D^{2} v(x) \geq 0$ for all $x \in[-\delta, \delta]$. Suppose $v(-\delta)>-\delta$. Then there exists a constant $c$ which we can take to be $\frac{16}{9}$ such that for every $N>0$ which satisfies

$$
v^{i}([-\delta, v(-\delta)]) \subseteq[-\delta, \delta] \quad \text { for } 0 \leq i \leq N
$$

we have for any $b_{1}, b_{2} \in[-\delta, v(-\delta)]$

$$
\frac{1}{c} \leq \frac{D\left(v^{N}\right)\left(b_{1}\right)}{D\left(v^{N}\right)\left(b_{2}\right)} \leq c .
$$

Proof. First suppose $b_{1}<b_{2}$. Since $[-\delta, v(-\delta)]$ is a fundamental interval for $v$ and $D v>0$ on $[-\delta, \delta]$ it follows that $v^{i}\left(b_{1}\right)<v^{i}\left(b_{2}\right)$ and $v^{i}\left(b_{2}\right)<v^{i+1}\left(b_{1}\right)$ for all $i, 0 \leq i \leq N$. Since $D^{2} v>0$ on $[-\delta, \delta]$ we see that

$$
D v\left(v^{i}\left(b_{1}\right)\right) \leq D v\left(\imath^{i}\left(b_{2}\right)\right)
$$


and

$$
D v\left(v^{i}\left(b_{2}\right)\right) \leq D v\left(v^{i+1}\left(b_{1}\right)\right)
$$

for all $i, 0 \leq i \leq N$. Now

$$
D\left(v^{N}\right)\left(b_{j}\right)=\prod_{i=0}^{N-1} D v\left(v^{i}\left(b_{j}\right)\right), \quad j=1,2
$$

so we have

$$
D\left(v^{N}\right)\left(b_{1}\right)=\prod_{i=0}^{N-1} D v\left(v^{i}\left(b_{1}\right)\right) \leq \prod_{i=0}^{N-1} D v\left(v^{i}\left(b_{2}\right)\right)=D\left(v^{N}\right)\left(b_{2}\right) .
$$

Also

$$
\begin{aligned}
D\left(v^{N}\right)\left(b_{2}\right) & =\prod_{i=0}^{N-1} D v\left(v^{i}\left(b_{2}\right)\right) \\
& =D v\left(v^{N-1}\left(b_{2}\right)\right) \prod_{i=0}^{N-2} D v\left(v^{i}\left(b_{2}\right)\right) \\
& \leq D v\left(v^{N-1}\left(b_{2}\right)\right) \prod_{i=0}^{N-2} D v\left(v^{i+1}\left(b_{1}\right)\right) \\
& =D v\left(v^{N-1}\left(b_{2}\right)\right) \prod_{i=1}^{N-1} D v\left(v^{i}\left(b_{1}\right)\right) \\
& =\frac{D v\left(v^{N-1}\left(b_{2}\right)\right)}{D v\left(b_{1}\right)} \prod_{i=0}^{N-1} D v\left(v^{i}\left(b_{1}\right)\right) \\
& =\frac{D v\left(v^{N-1}\left(b_{2}\right)\right)}{D v\left(b_{1}\right)} D\left(v^{N}\right)\left(b_{1}\right) .
\end{aligned}
$$

Hence

$$
\frac{D v\left(b_{1}\right)}{D v\left(v^{N-1}\left(b_{2}\right)\right)} \leq \frac{D\left(v^{N}\right)\left(b_{1}\right)}{D\left(v^{N}\right)\left(b_{2}\right)} \leq 1
$$

so

$$
\frac{9}{16} \leq \frac{D\left(v^{N}\right)\left(b_{1}\right)}{D\left(v^{N}\right)\left(b_{2}\right)} \leq 1 .
$$

Similarly, if $b_{1}>b_{2}$ we obtain

$$
1 \leq \frac{D\left(v^{N}\right)\left(b_{1}\right)}{D\left(v^{N}\right)\left(b_{2}\right)} \leq \frac{16}{9} .
$$

Combining these inequalities we complete the proof of the lemma.

Notation. Suppose $h: A \rightarrow A, I, J$ and $U$ are as in the definition of close-to-connection and in the remark following the definition. Fix intervals $I_{0} \subseteq J_{0} \subseteq I$ such that neither $I_{0}$ nor $J_{0}$ contains either end point of $J_{0}$ nor $I$, respectively (see figure 10). Let $W$ be a neighbourhood of the $\bigcup_{\zeta \in[0,1]} \gamma_{\xi}(\mathbb{T})$ given in condition (f) of the definition above.

LeMMA 11. Suppose $h: A \rightarrow A$ is $\left(\delta, m_{0}\right)$-close-to-connection with respect to $I, J$ and $U$ for $\alpha_{1}, \alpha_{2}$. Let $p / q$ be the rotation number of the periodic orbit of $h$. Then for any 


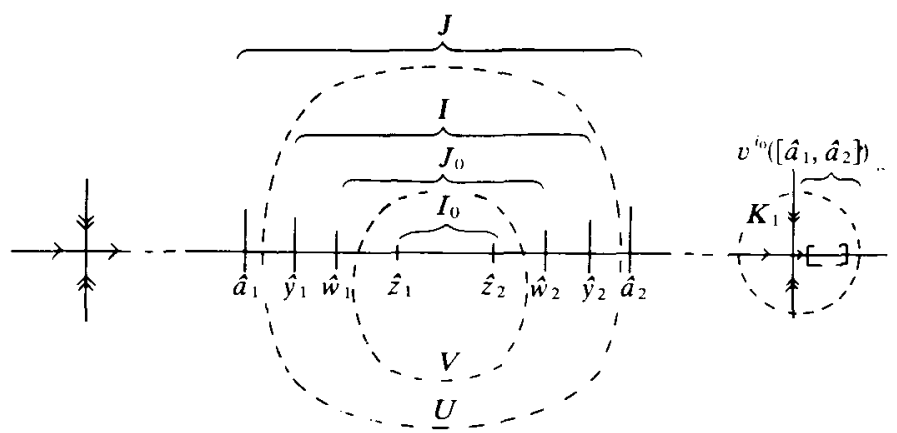

FIGURE 10

$\delta_{1}, \delta_{2}>0$ and neighbourhood $V$ of $I_{0}$ with $V \subseteq U \cap W, V$ bounded away from $\gamma(\mathbb{J}) \sim J_{0}$, there exists a $C^{\infty}$ diffeomorphism $g: A \rightarrow A$ such that

$$
\|g, h\|_{C^{m_{0}}<2 \delta}
$$

and $g$ is $\left(\delta_{1}, m_{0}+1\right)$-close-to-connection with respect to $I_{0}, J_{0}$ and $V$ for $\alpha_{1}, \alpha_{2}$. Moreover, $g$ may be chosen so that the rotation number $p_{0} / q_{0}$ of the periodic orbit of $g$ satisfies

$$
0<\left|\frac{p}{q}-\frac{p_{0}}{q_{0}}\right|<\delta_{2} .
$$

Proof. Let $h_{\zeta}: A \rightarrow A, \zeta \in[0,1]$ denote the one parameter family of maps associated with $h$. Since $h_{\zeta}$ is continuous in $\zeta \in[0,1]$ in the $C^{m_{0}+1}$ topology, we may fix $\zeta_{1} \in[0,1)$ sufficiently close to 1 so that $h_{\zeta}$ is $\left(\delta_{1} / 2, m_{0}+1\right)$-close-to-connection with respect to $I_{0}, J_{0}$ and $V$ for $\alpha_{1}, \alpha_{2}$ for any $\zeta \in\left[\zeta_{1}, 1\right)$.

Let $(\hat{r}, \hat{\theta})$ denote the $h_{\zeta_{1}}$-coordinate system (e.g. we may consider $\hat{\theta}$ a function of $(r, \theta), \hat{\theta}=\hat{\theta}(r, \theta))$. We assume (by rigidly rotating the $(\hat{r}, \hat{\theta})$ coordinates if necessary) that $\hat{\theta}\left(r_{2}, \theta_{2}\right)=\theta_{2}$. In these coordinates $h_{\zeta_{1}}$ may be written

$$
h_{\zeta_{1}}:(\hat{r}, \hat{\theta}) \rightarrow(k(\hat{r}, \hat{\theta}), v(\hat{\theta}))
$$

where $v: \mathbb{J} \rightarrow \mathbb{\pi}$ is a $C^{\infty}$ diffeomorphism with a single periodic orbit which contains the point $\theta_{2}$. Fix a neighbourhood $K_{1}$ of $\left(r_{2}, \theta_{2}\right)$ in $A$ so that

$$
\begin{gathered}
K_{1} \cap U=\varnothing, \\
K_{1} \cap\left\{\left(r_{i}, \theta_{i}\right): i=1, \ldots, q\right\}=\left\{\left(r_{2}, \theta_{2}\right)\right\}, \\
K_{1} \cap \gamma(\mathbb{T}) \text { is an interval }
\end{gathered}
$$

and

$$
\frac{4}{3}>D\left(v^{q}\right)(\hat{\theta})>\frac{3}{4}, \quad D^{2}\left(v^{q}\right)(\hat{\theta}) \neq 0
$$

for all $\hat{\theta}$ such that $(\hat{r}, \hat{\theta}) \in K_{1}$ for some $\hat{r}$. We may assume $D^{2}\left(v^{q}\right)(\hat{\theta})>0$ on $K_{1}$ without loss of generality. 
Fix numbers $\hat{a}_{i}, \hat{y}_{i}, \hat{w}_{i}$ and $\hat{z}_{i}$, with $i=1,2$ so that

$$
\begin{aligned}
J & =\left\{(\hat{r}, \hat{\theta}): \hat{r}=0, \hat{a}_{1} \leq \hat{\theta} \leq \hat{a}_{2}\right\} \\
I & =\left\{(\hat{r}, \hat{\theta}): \hat{r}=0, \hat{y}_{1} \leq \hat{\theta} \leq \hat{y}_{2}\right\} \\
J_{0} & =\left\{(\hat{r}, \hat{\theta}): \hat{r}=0, \hat{w}_{1}<\hat{\theta}<\hat{w}_{2}\right\} \\
I_{0} & =\left\{(\hat{r}, \hat{\theta}): \hat{r}=0, \hat{z}_{1}<\hat{\theta}<\hat{z}_{2}\right\}
\end{aligned}
$$

and hence $\hat{a}_{1}<\hat{y}_{1}<\hat{w}_{1}<\hat{z}_{1}<\hat{z}_{2}<\hat{w}_{2}<\hat{y}_{2}<\hat{a}_{2}<\hat{\theta}_{2}$ (see figure 10).

Let $\hat{K}_{1}=\left\{\hat{\theta}:(\hat{r}, \hat{\theta}) \in \gamma(\mathbb{T}) \cap K_{1}\right.$ for some $\left.\hat{r}\right\}$. Fix $i_{0}<0$ so that

$$
v^{i_{0}}\left(\left[\hat{a}_{1}, \hat{a}_{2}\right]\right) \subseteq \hat{K}_{1} \text {. }
$$

The set $\hat{K}_{1} \sim\left\{\theta_{2}\right\}$ is disconnected with

$$
v^{i_{0}}\left(\left[\hat{a}_{1}, \hat{a}_{2}\right]\right)
$$

in one component and

$$
\bigcup_{n>0}\left(v^{q}\right)^{n}\left(\left[\hat{a}_{1}, \hat{a}_{2}\right]\right) \cap \hat{K}_{1}
$$

in the other.

By lemma 10 there exists a constant $c_{2}>0$ such that

$$
c_{2}<\frac{v^{i_{0}-n q}\left(\hat{y}_{2}\right)-v^{i_{0}-n q}\left(\hat{w}_{2}\right)}{v^{i_{0}-n q}\left(\hat{a}_{2}\right)-v^{i_{0}-n q}\left(\hat{a}_{1}\right)}
$$

for all $n \geq 0$.

For $\zeta \in\left[\zeta_{1}, 1\right)$ let $(\tilde{r}, \tilde{\theta})$ denote the $h_{\zeta}$-coordinates (where we suppress the dependence of $\tilde{r}$ and $\tilde{\theta}$ on $\zeta)$, i.e. $\tilde{r}=\tilde{r}(\hat{r}, \hat{\theta})$ and $\tilde{\theta}=\tilde{\theta}(\hat{r}, \hat{\theta})$. We assume $\tilde{\theta}\left(0, g_{2}\right)=\theta_{2}$ and we let

$$
\tilde{a}_{i}=\tilde{\theta}\left(0, \hat{a}_{i}\right), \quad \tilde{y}_{i}=\tilde{\theta}\left(0, \hat{y}_{i}\right), \quad \tilde{w}_{i}=\tilde{\theta}\left(0, \hat{w}_{i}\right), \quad \tilde{z}_{i}=\tilde{\theta}\left(0, \hat{z}_{i}\right),
$$

for $i=1,2$. Writing

$$
h_{\zeta}:(\tilde{r}, \tilde{\theta}) \rightarrow\left(k_{1}(\tilde{r}, \tilde{\theta}), u(\tilde{\theta})\right)
$$

where we suppress the dependence of $k_{1}$ and $u$ on $\zeta$, we have, again by lemma 10 ,

$$
\frac{\left|u^{i q}\left(\tilde{y}_{2}\right)-u^{i q}\left(\tilde{y}_{1}\right)\right|}{\left|u^{i q}\left(\tilde{a}_{2}\right)-u^{i q}\left(\tilde{a}_{1}\right)\right|} \rightarrow 0
$$

uniformly in $i>0$ as $\zeta \rightarrow 1$. Hence there exists $\zeta_{2} \in\left[\zeta_{1}, 1\right)$ such that for all $\zeta \in\left[\zeta_{2}, 1\right)$

$$
\frac{\left|u^{i q}\left(\tilde{y}_{2}\right)-u^{i q}\left(\tilde{y}_{1}\right)\right|}{\left|u^{i q}\left(\tilde{a}_{2}\right)-u^{i q}\left(\tilde{a}_{1}\right)\right|}<\frac{c_{2}}{16}
$$

for all $i>0$.

Fix $\zeta_{3} \in\left[\zeta_{2}, 1\right)$ and fix a neighbourhood $K$ of $\left\{\left(r_{2}, \theta_{2}\right)\right\}$ such that $K \subseteq K_{1}$,

$$
\frac{1}{2}<\left|\begin{array}{ll}
\frac{\partial \tilde{r}}{\partial \hat{r}} & \frac{\partial \tilde{r}}{\partial \hat{\theta}} \\
\frac{\partial \tilde{\theta}}{\partial \hat{r}} & \frac{\partial \tilde{\theta}}{\partial \hat{\theta}}
\end{array}\right|<2, \quad \text { on } K,
$$

and $K \cap \gamma_{\zeta_{3}}(\mathbb{T})$ is an interval. Fix $N_{1}>0$ so large the

$$
u^{i_{0}-n q}\left(\left[\tilde{a}_{1}, \tilde{a}_{2}\right]\right) \subseteq \tilde{K}
$$


and

$$
u^{n q}\left(\left[\tilde{a}_{1}, \tilde{a}_{2}\right]\right) \subseteq \tilde{K}
$$

whenever $n \geq N_{1}$, where $\tilde{K}=\{\tilde{\theta} \in \mathbb{J}:(\tilde{r}, \tilde{\theta}) \in K$ for some $\tilde{r}\}$. Then for $n \geq N_{1}$

$$
\frac{\left|u^{i_{0}-n q}\left(\tilde{y}_{2}\right)-u^{i_{0}-n q}\left(\tilde{w}_{2}\right)\right|}{\left|u^{i_{0}-n q}\left(\tilde{a}_{2}\right)-u^{i_{0}-n q}\left(\tilde{a}_{1}\right)\right|}>\frac{c_{2}}{4},
$$

(see figure 11).

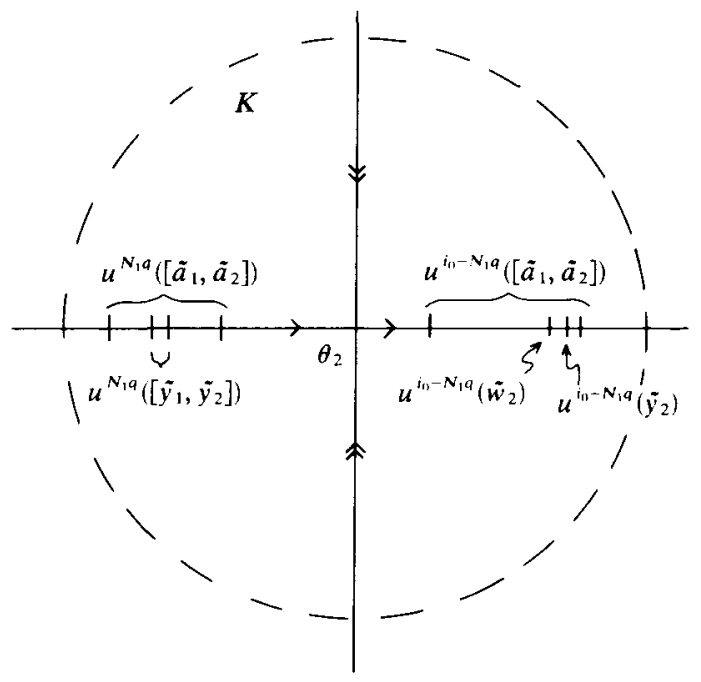

FIGURE 11

Next we wish to perturb $u$ to form a new map $\bar{u}: \mathbb{J} \rightarrow \mathbb{T}$ which has a single periodic orbit with rotation number $p_{0} / q_{0}$ satisfying

$$
0<\left|\frac{p}{q}-\frac{p_{0}}{q_{0}}\right|<\delta_{2}
$$

and such that $J_{0}$ is contained in a fundamental interval of $\bar{u}^{q_{0}}$.

Let $\psi: \mathbb{R} \rightarrow \mathbb{R}$ be a $C^{\infty}$ bump function with support in $\tilde{K}, 1 \geq \psi \geq 0, \psi\left(\theta_{2}\right)=1$. For each $\alpha>0$ let $u_{\alpha}: \mathbb{T} \rightarrow \mathbb{T}$ be the map given on $[0,2 \pi)$ by

$$
u_{\alpha}(\tilde{\theta})=u(\tilde{\theta})+\alpha \psi(\tilde{\theta}) \text {. }
$$

Fix $\beta_{1}>0$ so that whenever $0<\alpha<\beta_{1}, u_{\alpha}$ is a diffeomorphism and $u_{\alpha}$ satisfies (5.1) for $i=N_{1},(5.2)$ for $n=N_{1}$ and

$$
0<\left|\operatorname{rot}\left(u_{\alpha}\right)-\frac{p}{q}\right|<\delta_{2}, \quad \frac{4}{3}>D u_{\alpha}^{q}>\frac{3}{4}, \quad D^{2} u_{\alpha}^{q}>0,
$$

on $\tilde{K}$.

By lemma 10 and the continuity of $u_{\alpha}$ in $\alpha$, there exists a sequence $\left\{\alpha_{n}\right\}_{n=1}^{\infty}$ such that $\alpha_{n} \downarrow 0,0<\alpha_{1}<\beta_{1}$, and for each $n$ there exists $M_{n}>N_{1}$ such that for $N_{1} \leq i<M_{n}$, and $u_{\alpha_{n}}^{i q}\left(\left[\tilde{a}_{1}, \tilde{a}_{2}\right]\right) \subseteq \tilde{K}$,

$$
\left(u_{\alpha_{n}}\right)^{M_{n} q}\left(\left[\tilde{y}_{1}, \tilde{y}_{2}\right]\right) \subseteq\left(u_{\alpha_{n}}^{i_{0}-N_{1} q}\left(\tilde{w}_{2}\right), u_{\alpha_{n}}^{i_{0}-N_{1} q}\left(\tilde{y}_{2}\right)\right)
$$


Hence $u_{\alpha_{n}}$ has a periodic orbit with some period $s_{n}$ and $J_{0}$ is contained in a fundamental interval of $\left(u_{\alpha_{n}}\right)^{s_{n}}$. For each $n$, let $\nu_{n}$ denote the smallest positive number such that

$$
u_{\alpha_{n}}+\nu_{n}: \mathbb{T} \rightarrow \mathbb{T}
$$

has a single periodic orbit with period $s_{n}$. Then $J_{0}$ is contained in a fundamental interval of

$$
\left(u_{\alpha_{n}}+\nu_{n}\right)^{s_{n}}
$$

and since $\alpha_{n} \rightarrow 0$ as $u \rightarrow \infty$ it follows that $\nu_{n} \rightarrow 0$ as $n \rightarrow \infty$. Define

$$
\begin{aligned}
& g_{n}: A \rightarrow A \\
& g_{n}:(\tilde{r}, \tilde{\theta}) \rightarrow\left(k_{1}(\tilde{r}, \tilde{\theta}), u_{\alpha_{n}}(\tilde{\theta})+\nu_{n}\right) .
\end{aligned}
$$

Note that for each $n,\left.g_{n}\right|_{V}=\left.h_{\zeta_{3}}\right|_{V}$ and the invariant circle (as a set) and invariant foliation of $A$ are the same for $g_{n}$ as for $h_{\zeta_{3}}$, so $g_{n}$ satisfies conditions (1-12).

Let $\left(h_{\zeta_{3}}\right)_{\kappa}: A \rightarrow A, \kappa \in[0,1]$, be the one-parameter family of maps associated with $h_{\zeta_{3}}$ by which it is $\left(\delta_{1}, 12, m_{0}+1\right)$-close-to-connection with respect to $I_{0}, J_{0}$ and $V$ for $\alpha_{1}, \alpha_{2}$. Let

$$
\left(g_{n}\right)_{\kappa}=\left\{\begin{array}{l}
g_{n} \text { on } A \sim V \\
\left(h_{\zeta_{3}}\right)_{\kappa} \text { on } V
\end{array} \quad \text { for } \kappa \in[0,1] .\right.
$$

Let $\bar{\gamma}: \mathbb{T} \rightarrow A$ be the invariant circle for $\left(g_{n}\right)_{\kappa}$ (we suppress the dependence of $\bar{\gamma}$ on $\kappa, n)$ and note that $J_{0} \subseteq \bar{\gamma}(\mathbb{T})$ for all $\kappa \in[0,1]$. By choosing $n$ sufficiently large, we may fix a neighbourhood $W_{0}$ of

$$
\bigcup_{\kappa \in[0,1]} \bigcup_{i>0}^{\infty}\left(\left(g_{n}\right)_{\kappa}^{i}\left(J_{0}\right)\right)
$$

such that

$$
W_{0} \cap V=\varnothing,
$$

so there is a neighbourhood of $\bigcup_{\kappa \in[0,1]}\left(g_{n}\right)_{\kappa}^{q}\left(J_{0}\right)$ in which the $\left(g_{n}\right)_{\kappa}$-invariant foliation is independent of $\kappa$. Hence, fixing $n$ sufficiently large, we see that $g_{n}=g$ is $\left(\delta_{1}, m_{0}+1\right)$-close-to-connection with respect to $I_{0}, J_{0}$ and $V$ for $\alpha_{1}, \alpha_{2}$, and the proof of the lemma is complete.

Now to construct the desired sequence $\left\{h_{n}: A \rightarrow A\right\}_{n=1}^{\infty}$ of diffeomorphisms we let $h_{1}=f$ be the map of the first part of this section. Let $I_{1}$ and $J_{1}$ be the intervals associated with $f$ also as defined in the first part of this section. Define a sequence of intervals $I_{n}, J_{n}$ such that

$$
J_{1} \supseteq I_{1} \supseteq J_{2} \supseteq I_{2} \supseteq \cdots
$$

each interval containing neither of the end points of the interval before it in the sequence and

$$
\bigcap_{i=1}^{\infty} I_{i}=I \neq \varnothing
$$

and $I$ is not a singleton. Fix $\delta_{1}$ so that $f=h_{1}$ is $\left(\delta_{1}, 2\right)$-close-to-connection with respect to $I_{1}, J_{1}$ for some neighbourhood $V$ and constants $\alpha_{1}, \alpha_{2}$. Choose $\left\{\delta_{n}\right\}_{n=2}^{\infty}$ 
so that

$$
\sum_{n=2}^{\infty} \delta_{n}=\frac{\varepsilon}{2}<\infty
$$

and every $C^{\infty}$ map in an $2 \varepsilon$-neighbourhood in the $C^{1}$ topology of $f_{\zeta}$, for any $\zeta \in[0,1]$, is a diffeomorphism of $\boldsymbol{A}$ into $\boldsymbol{A}$.

LEMMA 12. Suppose we have chosen $h_{i}: A \rightarrow A, i=1, \ldots, n$ such that for each $i=1, \ldots, n$ there exists a neighbourhood $V_{i}, i=1, \ldots, n$ such that

(i) $h_{i}$ is $\left(\delta_{i}, i+1\right)$-close-to-connection with respect to $I_{i}, J_{i}$ and $V_{i}$ for $\alpha_{1}, \alpha_{2}$;

(ii) if $p_{j} / q_{j}$ is the rotation number of $h_{j}, j=1, \ldots, n$ then

$$
0<\left|\frac{p_{i}}{c_{i}}-\frac{p_{j}}{q_{j}}\right|<\frac{1}{10 q_{j}^{2}}
$$

whenever $j<i$;

(iii) let $\gamma_{i}: \mathbb{T} \rightarrow A$ be the invariant circle for $h_{i}$; then there exists a neighbourhood $W_{i}$ of $\gamma_{i}(\mathbb{T})$ such that

$$
W_{i+1} \subseteq h_{i}\left(W_{i}\right) \subseteq W_{i}, \quad V_{i+1} \subseteq W_{i+1},
$$

and for each $z_{0} \in W_{i}$

$$
\inf _{z \in \gamma_{i}(\mathbb{U})}\left\|z, z_{0}\right\|<\delta_{i}
$$

(iv) $\left\|h_{i}, h_{i+1}\right\|_{C^{i}}<2 \delta_{i}$.

Then there exists a map $h_{n+1}: A \rightarrow A$ such that (i)-(iv) are satisfied for $i=1, \ldots, n+1$.

Proof. The proof is immediate from lemma 11.

Remark. Condition (ii) above is needed only to ensure that $\lim _{i \rightarrow \infty} p_{i} / q_{i}$ is irrational. We could replace this estimate by much more severe estimates requiring the $\lim _{i \rightarrow \infty} p_{i} / q_{i}$ to be a Louiville number (well-approximable by rationals).

Since the induction step requires us to make small perturbations we can not gain any information about how poorly-approximable by rationals the rotation number of our example might be.

Proof of theorem 3. By induction and lemma 12 there exists a sequence $\left\{h_{n}: A \rightarrow\right.$ $A\}_{n=1}^{\infty}$ of $C^{\infty}$ diffeomorphisms satisfying (i)-(iv) of lemma 12 . By condition (iv) the limit $h=\lim _{n \rightarrow \infty} h_{n}$ exists and is a $C^{\infty}$ diffeomorphism. If $\gamma_{n}: \mathbb{T} \rightarrow A$ denotes the invariant circle for $h_{n}$ then by conditions (i) and (iii) there is a curve $\gamma: \mathbb{T} \rightarrow A$ such that $\gamma=\lim _{n \rightarrow \infty} \gamma_{n}$ and $\gamma$ satisfies condition (7) above (with $\left(\alpha_{1}, \alpha_{2}\right)$ replacing $\left[\alpha_{1}, \alpha_{2}\right]$ ). By condition (iii) the set $\gamma(\mathbb{J})$ is the attractor for the attractor block $A$. Since $I=\bigcap_{i=1}^{\infty} I_{i}$ is a subset of $\gamma_{j}$ for each $j=1,2, \ldots$, we see that $I \in \gamma(\mathbb{T})$. Let $z_{n}$ be a point on the periodic orbit of $h_{n}$. Then

$$
h_{n}^{i}\left(z_{n}\right) \notin I \quad \text { for all } i>0 \text {. }
$$

iNence if $z_{0}$ is the limit of a convergent subsequence of $\left\{z_{n}\right\}_{n=1}^{\infty}$ then for any $\bar{I} \subseteq I$, $\bar{I}$ a non empty, non-singleton interval not containing either end point of $I$ then

$$
h^{i}\left(z_{0}\right) \notin \bar{I} \quad \text { for all } i \geq 0 \text {. }
$$


Hence $\left.h\right|_{\gamma(\mathbb{T})}$ has orbits which are not dense. Since

$$
\left.h\right|_{\gamma(\pi)}=\left.\lim _{n \rightarrow \infty} h_{n}\right|_{\gamma_{n}(\pi)}
$$

we see that the rotation number of $h_{\gamma(\mathbb{T})}$ is

$$
\rho=\lim _{n \rightarrow \infty} \frac{p_{n}}{q_{n}} .
$$

By condition (ii) of lemma 12 we see that $\rho$ has infinitely many rational approximates, so $\rho$ is irrational. Hence $h$ is the required map and the proof of the theorem is complete.

Remarks. (1) Again we note that the only control we have on the rotation number of the example above is to make the rotation number as well-approximable by rationals as we wish and close to any given rational. We do not know if such examples exist for arbitrary irrational rotation numbers.

(2) The starting point of the above construction is a map with an interval of heteroclinic points in the projective bundle, or, in other terms, an interval on the invariant circle lies on a leaf of an invariant foliation near a periodic orbit. It has been observed numerically (see [2]) that points of tangency of this type between an invariant foliation and an invariant circle occur even for two-parameter families of quadratic maps of the plane. By a $C^{1}$ perturbation we can turn this point of tangency into an interval of tangency. Then a $C^{\infty}$ small perturbation leads to examples as in theorem 3. Other than this, we have no information on how common such maps are in the space of $C^{\infty}$ diffeomorphisms of the annulus. In particular we do not know if ' $C C^{\infty}$ ' can be replaced by 'analytic' in theorem 3.

This paper is the author's Ph.D thesis, written at the University of Minnesota under the direction of Richard McGehee. Without his patient assistance this work would hardly have been started, much less completed. The author would also like to thank R. McGehee and D. G. Aronson for the opportunity of serving as research assistant in the Dynamical Systems Laboratory in the University of Minnesota.

\section{REFERENCES}

[1] V. I. Arnold. Loss of stability of self-oscillations close to resonance and versal deformations of equivariant vector fields. Functional Analysis and its Applications 11 (2) (1977), 1-10.

[2] D. G. Aronson, M. A. Chory, G. R. Hall \& R. P. McGehee. Bifurcation from an invariant circle for two parameter families of maps of the plane: a computer-assisted study. Comm. Math. Phys. 83 (1982), 303-354.

[3] L. B. Bushard. Periodic solutions and locking-in on the periodic surface. J. Non-linear Mechanics 8 (1973), 129-141.

[4] C. Conley. Isolated Invariant Sets and the Morse Index. CBMS Regional Conference Series in Mathematics. No. 38. American Mathematical Society: Providence, 1978.

[5] J. Curry \& J. Yorke. A transition from Hopf bifurcation to chaos: computer assisted experiments on maps in $\mathbb{B}^{2}$. The Structure of Attractors in Dynamical Systems (ed. N. G. Markley, J. C. Martin, W. Perrizo), Lecture Notes in Mathematics 668, pp 48-68. Springer-Verlag: Berlin, 1978.

[6] A. Denjoy. Sur les courbes definies par les equations differentialles a la surface du tore, J. de Math. Pures et Appliquees, 11 (1932), 33-375. 
[7] N. Fenichel. Persistance and smoothness of invariant manifolds for flows. Indiana University Mathematics Journal 21 (3) (1971), 193-226.

[8] N. Fenichel. The orbit structure of the Hopf bifurcation problem. J. Diff. Eq. 11 (2) (1975), 308-328.

[9] M. Handel. A pathological area preserving $C^{\infty}$ diffeomorphism of the plane. Proc. Amer. Math. Soc. (To appear.)

[10] J. Harrison. Denjoy fractals in the plane. Preprint.

[11] J. Harrison. A $C^{2}$ counter example to the Seifert conjecture. Preprint.

[12] M. R. Herman. Sur la conjugaison differentialles des diffeomorphisms du circle a des rotation. Publ. Math. IHES 49 (1979), 5-233.

[13] M. R. Herman. Contre-exemple de Denjoy et contre-exemples de class $C^{3-\varepsilon}$ au theoreme des courbes invariantes un nombre de rotation fixe. (To appear as chapter III of Introduction a l'etude des courbes invariante des diffeomorphism de l'annneau.)

[14] M. W. Hirsch, C. Pugh \& M. Shub. Invariant Manifolds. Lecture Notes in Mathematics 583. Springer-Verlag: Berlin, 1977.

[15] R. J. Knill. A $C^{\infty}$ flow on $S^{3}$ with a Denjoy minimal set. J. Diff. Geom. 16 (1982), 271-280.

[16] J. Marsden \& M. McCracken. The Hopf Bifurcation and its Applications. Notes in Applied Science 19, Springer-Verlag: Berlin, 1976.

[17] R. P. McGehee. The stable manifold theorem via an isolating block. Symposium on Ordinary Differential Equations (ed. W. A. Harris, Y. Sibuya), Lecture Notes in Mathematics 312, pp 135-144. Springer-Verlag: Berlin, 1972.

[18] M. Neimark. On some cases of dependence of periodic motion on parameters. Dokl. Akad. Nauk. SSSR 129 (1959), 736-739.

[19] H. Poincare. Les Methods Nouvelles de la Mechanic Celeste Vol. 3. Gauthiers-Verlag, 1899.

[20] D. Ruelle \& F. Takens. On the nature of turbulence. Comm. Math. Phys. 20 (1971), 126-137.

[21] R. Sacker. On invariant surfaces and bifurcation of periodic solutions of ordinary differential equations. Thesis, New York University, IMM-NYU (October 1964).

[22] J. F. Selgrade. Isolated invariant sets for flows on vector bundles. Trans. Amer. Math. Soc. 203 (1975), 359-390. 